Z Rheumatol 2022 $\cdot 81: 744-759$

https://doi.org/10.1007/s00393-021-01093-1

Angenommen: 17. August 2021

Online publiziert: 15 . Oktober 2021

() Der/die Autor(en) 2021

\section{Redaktion}

Ulf Müller-Ladner, Bad Nauheim

Uwe Lange, Bad Nauheim

\section{Zusatzmaterial online}

Zusätzliche Informationen sind in der Online-Version dieses Artikels (https:// doi.org/10.1007/s00393-021-01093-1) enthalten.

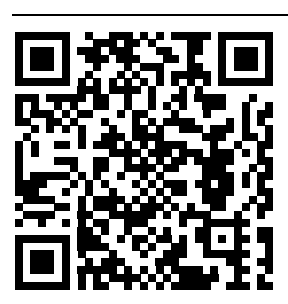

QR-Code scannen \& Beitrag online lesen

\title{
Entwicklung von Qualitätsstandards für die Versorgung von Patient*innen mit rheumatoider Arthritis zur Anwendung in Deutschland
}

U. Kiltz ${ }^{1,2} \cdot$ V. Buschhorn-Milberger ${ }^{1} \cdot$ K. Albrecht ${ }^{3} \cdot$ H.-J. Lakomek ${ }^{4} \cdot$ H.-M. Lorenz ${ }^{5}$ M. Rudwaleit ${ }^{6} \cdot$ M. Schneider $\cdot$ H. Schulze-Koops ${ }^{8} \cdot$ M. Aringer ${ }^{9} \cdot$ M. I. Hasenbring ${ }^{10}$. P. Herzer ${ }^{11} \cdot$ U. von Hinüber ${ }^{12} \cdot K$. Krüger ${ }^{13} \cdot$ A. Lauterbach ${ }^{14} \cdot$ B. Manger ${ }^{15} \cdot$ R. Oltman ${ }^{16} \cdot$ F. Schuch ${ }^{17} \cdot$ R. Schmale-Grede ${ }^{18} \cdot$ S. Späthling-Mestekemper ${ }^{19} \cdot$ S. Zinke ${ }^{20,21} \cdot$ J. Braun $^{1,2}$ ${ }^{1}$ Rheumazentrum Ruhrgebiet, Herne, Deutschland; ${ }^{2}$ Ruhr-Universität Bochum, Bochum, Deutschland; ${ }^{3}$ Programmbereich Epidemiologie, Deutsches Rheuma-Forschungszentrum (DRFZ), Berlin, Deutschland; ${ }^{4}$ Johannes-Wesling-Klinikum Minden, Universitätsklinik für Geriatrie, Minden, Deutschland; ${ }^{5}$ Sektion Rheumatologie, Medizinische Klinik V, Universitätsklinikum Heidelberg, Universität Heidelberg, Heidelberg, Deutschland; ${ }^{6}$ Universitätsklinik für Innere Medizin und Rheumatologie, Klinikum Bielefeld Rosenhöhe, Universität Bielefeld, Bielefeld, Deutschland; ' ${ }^{7}$ Poliklinik, Funktionsbereich und Hiller Forschungszentrum für Rheumatologie, Universitätsklinikum Düsseldorf, Heinrich-Heine-Universität Düsseldorf, Düsseldorf, Deutschland; ${ }^{8}$ Sektion Rheumatologie und Klinische Immunologie, Medizinische Klinik und Poliklinik IV, LMU-Klinikum München, Ludwig-Maximilians-Universität München, München, Deutschland; ${ }^{9}$ Medizinische Klinik und Poliklinik III, Rheumatologie, Universitätsklinikum Carl Gustav Carus, Technische Universität Dresden, Dresden, Deutschland; ${ }^{10}$ Abteilung für Medizinische Psychologie und Medizinische Soziologie, Ruhr-Universität Bochum, Bochum, Deutschland; "Medicover München MVZ, München, Deutschland; ${ }^{12}$ Praxis für Rheumatologie und Osteologie, Hildesheim, Deutschland; ${ }^{13}$ Rheumatologisches Praxiszentrum St. Bonifatius, München, Deutschland; ${ }^{14}$ Physiotherapieschule Friedrichsheim, Friedrichsheim, Deutschland; ${ }^{15}$ Medizinische Klinik 3 Rheumatologie und Immunologie, Universitätsklinikum, Friedrich-Alexander-Universität Erlangen/Nürnberg, Erlangen, Deutschland; ${ }^{16}$ Hochschule für Gesundheit Bochum, Bochum, Deutschland; ${ }^{17}$ Rheumatologische Schwerpunktpraxis Erlangen, Erlangen, Deutschland; ${ }^{18}$ Deutsche Rheuma-Liga Bundesverband e. V., Bonn, Deutschland; ${ }^{19}$ Rheumapraxis München, München, Deutschland; ${ }^{20}$ Rheumatologische Schwerpunktpraxis Zinke, Berlin, Deutschland; ${ }^{21}$ Bundesverband Deutscher Rheumatologen e.V. (BDRh), Grünwald, Deutschland
Die rheumatoide Arthritis (RA) ist eine meist die peripheren Gelenke von Händen und Füßen betreffende entzündlich rheumatische Erkrankung, welche unbehandelt oft zu Osteodestruktionen durch Gelenkerosionen und Gelenkfehlstellungen führt [1]. Initial treten in der Regel symmetrische Schwellungen von Gelenken zusammen mit länger anhaltender Morgensteifigkeit auf. Auch in anderen Organen wie der Lunge oder den Blutgefäßen kann es zu Manifestationen der RA kommen.

Die Versorgungsqualität, nicht nur von Patient*innen mit RA, weist trotz Entwicklung und regelmäßiger Aktualisierung evidenzbasierter Leitlinien (LL) durch die Fachgesellschaft DGRh [2,3] nach wie vor in einigen Bereichen Defizite auf. So fand sich v.a. bei älteren RA-Patient*innen, bei RA-Patient*innen ohne laborchemischen Nachweis von Rheumafaktor sowie bei RA-Patient*innen ohne fachärztliche Betreuung eine Unterversorgung mit DMARDs, insbesondere mit Biologika (bDMARDs) [4-7]. Zusätzlich konnten eine Unterversorgung in der Verordnung von Physiotherapie [8, 9], eine eher inkonsequent angewendete Treat-to-targetStrategie [5], zu späte oder fehlende Überweisungen von RA-Verdachtsfällen [10] und die nicht seltene Versorgung durch Ärzt*innen ohne internistisch-rheumatologische Facharztkompetenz aufgezeigt werden [4]. Darüber hinaus gibt es Hinweise auf eine unzureichende Versorgung von Patient*innen mit Komorbiditäten [11]. Auch zeigen sich eine Unterversorgung der Komorbidität Osteoporose 
[12] und unzureichende Präventivmaßnahmen, wie z.B. Impfungen [13, 14]. Außerdem werden RA-Patienten*innen, die im Pflegeheim leben, weniger häufig von rheumatologischen Fachärzt*innen betreut und erhalten demnach seltener DMARDs [15].

Dessen ungeachtet sind durchaus auch positive Entwicklungen zu verzeichnen wie etwa die Einführung der ambulanten spezialfachärztlichen Versorgung (ASV), die Einführung von Früharthritissprechstunden, die Anerkennung der rheumatologischen Fachassistenz (RFA) durch die Bundesärztekammer, das Patientenschulungs- und Informationsprogramm der DGRh [16] und nicht zuletzt das durch den Gemeinsamen Bundesauschuss beschlossene Disease-Management-Programm RA [17].

Das Ziel der Entwicklung von Qualitätsstandards (QS) ist es, bestehende Versorgungslücken zu identifizieren und dann den vorliegenden Problemen entsprechende standardisierte Messparameter für die Verbesserung der Qualität in den betroffenen Versorgungsbereichen vorzuschlagen.

Bislang gibt es zwar internationale QS, auf nationaler Ebene wurden aber bisher noch keine QS für die Versorgung von RA-Patient*innen generiert. International wurden einige z. T. sehr umfangreiche Pakete von Qualitätsstandards und/oder -indikatoren entwickelt. Während sich die adressierten Schlüsselbereiche zur Quali-

\section{Abkürzungen}

\begin{tabular}{|c|c|}
\hline $\mathrm{Ag}$ & Arbeitsgruppe \\
\hline ASV & $\begin{array}{l}\text { Ambulante spezialfachärztliche } \\
\text { Versorgung }\end{array}$ \\
\hline BDRh & $\begin{array}{l}\text { Berufsverband Deutscher Rheuma- } \\
\text { tologen }\end{array}$ \\
\hline DGRh & $\begin{array}{l}\text { Deutsche Gesellschaft für Rheuma- } \\
\text { tologie }\end{array}$ \\
\hline DMARD & $\begin{array}{l}\text { Disease-modifying anti-rheumatic } \\
\text { drug }\end{array}$ \\
\hline EULAR & $\begin{array}{l}\text { European League Against Rheuma- } \\
\text { tism }\end{array}$ \\
\hline$L L$ & Leitlinien \\
\hline NICE & $\begin{array}{l}\text { National Institute for Health and } \\
\text { Care }\end{array}$ \\
\hline NRS & Numerical rating scale \\
\hline$Q S$ & Qualitätsstandards \\
\hline$R A$ & Rheumatoide Arthritis \\
\hline$S L R$ & Systematische Literaturrecherche \\
\hline VRA & $\begin{array}{l}\text { Verband Rheumatologischer } \\
\text { Akutkliniken }\end{array}$ \\
\hline
\end{tabular}

Trotz einer qualitativ und strukturell guten Versorgung von Patient*innen mit rheumatoider Arthritis (RA) in Deutschland bestehen weiterhin potenziell behebbare Defizite in der Qualität der Versorgung. Aus diesem Grund hat die Deutsche Gesellschaft für Rheumatologie (DGRh) eine Expert*innengruppe, in der verschiedene Interessengruppen vertreten waren, beauftragt, nationale Qualitätsstandards (QS) mit dem Ziel zu entwickeln, die rheumatologische Versorgung von Patient*innen mit RA in Deutschland qualitativ zu verbessern. QS dienen der Festlegung und quantitativen Messung guter Versorgungsqualität unter dem Vorbehalt von Relevanz und Realisierbarkeit. Als Grundlage für die Entwicklung dienten die kürzlich publizierten Standards von NICE und ASAS und eine systematische Literatursuche. Insgesamt wurden 8 hiermit erstmals veröffentlichte QS konsentiert, die als Grundlage dienen können, die Versorgungsqualität von Patient*innen mit RA in Deutschland zu messen und weiter zu optimieren.

\section{Schlüsselwörter}

Rheumatoide Arthritis · Versorgungsqualität · Qualitätsstandards · Versorgungslücken

tätsverbesserung bei diesen Vorschlägen häufig ähneln und zum Teil überlappen, ist es im Rahmen der Entwicklung der QS zu sehr unterschiedlichen Vorgehensweisen gekommen, d.h. eine einheitliche Methodik wurde bislang nicht verwendet. Es gibt mehrere Modelle zur Entwicklung von QS [18]. In England hat sich das $\mathrm{Na-}$ tional Institute for Health and Care Excellence (NICE) auf eine Methodik geeinigt [19], auf die auch bereits an anderer Stelle [20] zurückgegriffen wurde. Allen Modellen ist gemeinsam, dass gute Versorgungsqualität auf Grundlage der Arbeiten von Donabedian definiert wird [21]. Dies beinhaltet, dass bei der Beurteilung von Versorgungsqualität neben Strukturund Prozessqualität auch Ergebnisqualität zu berücksichtigen ist. Während die Strukturqualität v.a. durch das Vorhandensein von definierten Strukturen wie etwa von geeigneten Ambulanzräumen, einem UItraschallgerät oder der Vorhaltung eines behindertengerechten Praxiszugangs gemessen wird, bedeutet Prozessqualität die Organisation bzw. den Ablauf eines Prozesses, wie z.B. die Terminplanung einer Sprechstunde oder die Verfügbarkeit von geeignetem Praxispersonal. Struktur- und Prozessqualität sind eine wichtige Grundlage für die Ergebnisqualität, die unter anderem den Therapierfolg abbildet, wobei z.B. die Anzahl von Patient*innen, die in einem definierten Zeitraum eine Remission erreichen, gemessen werden könnte [21].

Ein erstes Projekt zur Qualitätssicherung der Versorgung von rheumatischen Erkrankungen gibt es in Deutschland bereits seit geraumer Zeit in Form des OBRA ("outcome benchmarking“ in der rheumatologischen Akutversorgung) bzw. KOBRA(kontinuierliches Outcome-Benchmarking in rheumatologischen Akutkliniken)-Projekts, welches seit Jahren in mehreren Kliniken der akutstationären Versorgung durch den Verband Rheumatologischer Akutkliniken (VRA) in Kooperation mit dem Institut für angewandte Qualitätsförderung und Forschung im Gesundheitswesen (AQUA) erfolgreich durchgeführt wird $[22,23]$.

Hierfür wurden Qualitätsindikatoren definiert, um Versorgungsqualität und Patient*innenzufriedenheit zu messen und um zwischen verschiedenen Kliniken Vergleiche anstellen zu können (Benchmarking) - mit dem Ziel, die Versorgungsqualität zu verbessern. Die Daten werden in den Kliniken erhoben und vom unabhängigen AQUA-Institut analysiert und ausgewertet. Für die Teilnahme am Benchmarkingprozess erhalten die teilnehmenden Kliniken, wenn sie auch sonst die Qualitätsansprüche erfüllen [24], ein Zertifikat und Gütesiegel des VRA. Nachdem zunächst nur Patient*innen mit RA in das Projekt eingeschlossen wurden, wird inzwischen ein deutlich größeres Spektrum entzündlich rheumatischer Erkrankungen erfasst.

Die Deutsche Gesellschaft für Rheumatologie (DGRh) hat es sich zur Aufgabe gemacht, nationale QS zu formulieren, um die Versorgungsqualität von RA-Patient*innen in Deutschland messen und verbessern zu können. Dabei sollte der formulierte Qualitätsstandard ein wünschenswertes, 
Tab. 1 Phasen der Entwicklung von QS für RA

\begin{tabular}{|l|l|l|}
\hline Phase & Ziel & Methode \\
\hline 1 & $\begin{array}{l}\text { Teil 1: Identifikation von publizierten Qualitätsmessinstrumen- } \\
\text { ten für RA (bezogen auf Methodik und Inhalt) } \\
\text { Teil 2: Identifikation von relevanten Versorgungslücken }\end{array}$ & SLR \\
\hline 2 & $\begin{array}{l}\text { Identifikation der Schlüsselbereiche für die Qualitätsverbesse- } \\
\text { rung }\end{array}$ & $\begin{array}{l}\text { Virtuelles Treffen } \\
\text { rung }\end{array}$ \\
\hline 3 & Formulierung der Qualitätsstandards für RA & $\begin{array}{l}\text { Standardisierte } \\
\text { Online-Abfrage }\end{array}$ \\
\hline 4 & Konsentierung der Qualitätsstandards für RA & $\begin{array}{l}\text { Strukturierte } \\
\text { Konsensfindung, } \\
\text { virtuell }\end{array}$ \\
\hline 5 & & $\begin{array}{l}\text { Grad der Zustim- } \\
\text { mung, NRS 0-10 }\end{array}$ \\
\hline
\end{tabular}

jedoch auch realisierbares, Versorgungsziel repräsentieren. Das langfristige Ziel besteht darin, die Qualität in Deutschland durch messbare Qualitätskonstrukte zu dokumentieren und zu verbessern, um in $\mathrm{Zu}$ kunft ein hohes Maß an Versorgungsqualität von RA-Patient*innen gewährleisten zu können.

\section{Methoden}

Die Entwicklung der Qualitätsstandards für RA startete im September 2019 mit der Bildung eine Steuerungsgruppe mit ausgewählten Expert*innen mit Expertise in der Versorgung von $\mathrm{Pa}$ tient*innen mit RA (KA, JB, UK, HS-K, H-JL, H-ML, MR, MS). Die Steuerungsgruppe entschied sich nach eingehender Diskussion, alle in der Behandlung der RA involvierten Interessengruppen (ambulant und stationär tätige Rheumatolog*innen, Physiotherapeut*innen, Ergotherapeut*innen, Psycholog*innen, Patient*innenvertreter*innen) aktiv in die Entwicklung der QS für RA als Arbeitsgruppe (AG) einzubeziehen. Alle eingeladenen Vertreter (Physiotherapie vertreten durch AL, Ergotherapeuten durch RO, Psychologen durch $\mathrm{MIH}$, Patienten durch RSG) beteiligten sich aktiv an dem Entwicklungsprozess und waren stimmberechtigt. Nicht stimmberechtigt war VBM als Methodikerin. Die Steuerungsgruppe legte die Methodik und den Ablauf der Entwicklung der Qualitätsstandards in 5 Phasen fest (- Tab. 1).

Die Entwicklung der Qualitätsstandards für RA erfolgte parallel zu der Erstellung der Qualitätsstandards für die axiale Spondyloarthritis [25].
Phase 1 mit Erstellung der systematischen Literaturrecherche. Beide SLR wurden in PubMed und Cochrane für den Zeitraum 01.05.2005-01.05.2020 durchgeführt (Suchterms s. Online-Zusatzmaterial). Es wurde von der Steuerungsgruppe festgelegt, dass englisch- und deutschsprachige Publikationen als Vollpublikation eingeschlossen werden konnten. Die Suche zu Qualitätsmessinstrumenten für RA fokussierte auf die Identifikation von Methodik und Inhalt. Für die Selektion von Publikationen zu Versorgungslücken wurden folgende Einschlusskriterien festgelegt: Studientyp (kontrollierte Studien/ Kohortenstudien/Fall-Kontrollstudien mit einer Fallzahl $\geq 200$ Patient*innen) und qualitative Studien (ohne Teilnehmerbegrenzung).

Phase 2: Identifikation der Schlüsselbereiche für die Qualitätsverbesserung. Schlüsselbereiche sind Bereiche, in denen es Unterschiede in der Versorgung gibt, deren Verbesserung aber realistisch ist und auch quantitativ erfasst werden kann. Auf der Grundlage der SLR erfolgte die Identifikation von Versorgungslücken. Die Schlüsselbereiche sind unter folgenden 5 Aspekten diskutiert worden: (I) Validität (,Wie valide ist die wissenschaftliche Evidenz, dass es sich bei dem genannten Schlüsselbereich, um eine im klinischen Alltag relevante Domäne handelt?"), (II) Augenscheinvalidität (,Wie wahrscheinlich ist es, dass eine bessere Versorgungsleistung in dem genannten Schlüsselbereich ein qualitativ hochwertigeres Gesundheitssystem widerspiegelt?"), (III) Durchführbarkeit („Wie wahrscheinlich ist es, dass die für den
Schlüsselbereich erforderliche Information in Ihrem Gesundheitssystem verfügbar ist?"), (IV) Bedeutung: („Wie wichtig ist es, diesen Schlüsselbereich zu messen?") und (V) Wahrscheinlichkeit der Verwendung (,Wie wahrscheinlich ist es, dass Sie diesen Schlüsselbereich in Ihrer Praxis/Klinik verwenden oder zur Verwendung dieses Indikators ermutigen würden?"). Die AG diskutierte auf einem virtuellen Treffen die Versorgungslücken sehr intensiv und legte eine Liste mit Versorgungslücken an, die quantitativ erfasst werden können.

Phase 3: Priorisierung der Versorgungslücken. Die identifizierten Schlüsselbereiche wurden im Anschluss an das virtuelle Treffen per Online-Umfrage priorisiert. Ziel der Priorisierung war es, die relevanten Schlüsselbereiche zu identifizieren und die vorläufige Liste auf eine handhabbare finale Liste mit ca. 7 bis 10 Schlüsselbereichen für die Qualitätsverbesserung zu reduzieren. Die Priorisierung wurde erhoben, indem nach der Relevanz („Stimmen Sie zu, dass diese Domäne eine relevante Versorgungslücke in Deutschland darstellt?") und nach dem Grad der Zustimmung (numerische Rating-Skala 0-10, $10=$ höchste Zustimmung) gefragt wurde.

Phase 4: Formulierung der Qualitätsstandards für RA. Nach Festlegung der 8 Versorgungslücken erfolgte in einem virtuellen Treffen die Ausformulierung der Qualitätsstandards in Bezug auf Struktur und Prozessqualität. Ein Qualitätsstandard besteht aus 2 Bereichen: (I) der Kernaussage mit einer begleitenden Rationale, in der die Evidenz für die Aussage dargelegt wird, und (II) einem Messinstrument, in dem die Angaben zur Berechnung der Struktur- und Prozessqualität angegeben sind. Das Ziel der AG in dem virtuellen Meeting im Dezember 2020 war es, Kernaussagen zu erarbeiten und diese dann jeweils mit einer Rationale und Qualitätsmessinstrumenten zu unterfüttern. Die im Anschluss erfolgte schriftliche Ausformulierung der QS wurde mittels E-MailSchriftverkehr mehrfach überarbeitet.

Phase 5: Konsentierung der Qualitätsstandards. Nach Ausformulierung der Qualitätsstandards wurden die Teilnehmer der AG gebeten, in einer Online-Umfrage 


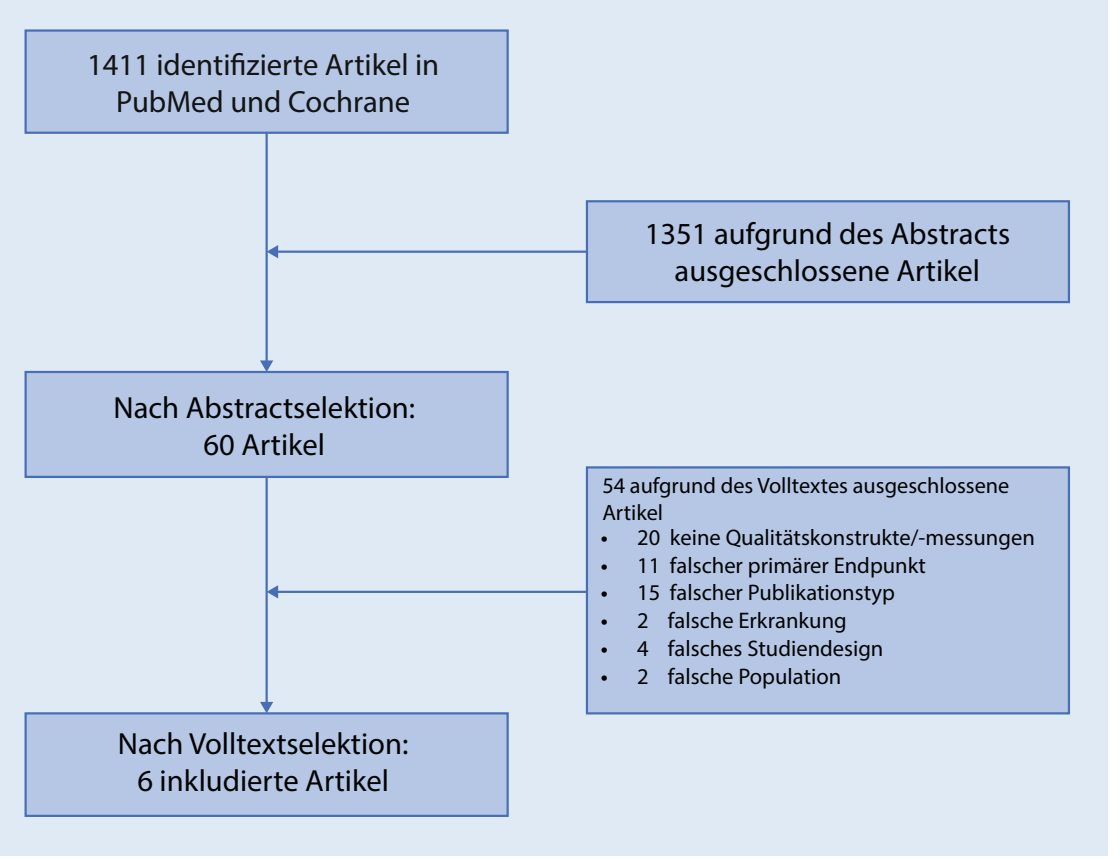

Abb. 1 ॥ Flussdiagramm der SLR der Phase 1a: Identifikation von etablierten Qualitätsstandards für RA

den Grad der Zustimmung (mittels NRS $0-10,10$ stimme voll zu) anzugeben.

\section{Ergebnisse}

\section{Erstellung der Qualitätsstandards}

Die Analyse der einzelnen Schritte ergab folgende Ergebnisse:

\section{Phase 1 mit Erstellung der systematischen Literaturrecherche} SLR Qualitätsmessinstrumente: Die einzelnen Schritte der SLR hinsichtlich bereits publizierter Qualitätsmessinstrumente listet - Abb. 1 auf. Insgesamt wurden 6 Publikationen zu Qualitätsmessinstrumenten der RA identifiziert [26-31] Die ersten QS wurden 2005 in England veröffentlicht [30], gefolgt von Publikationen in den USA und Europa [27, 31]. Drei weitere Artikel beziehen sich auf Arthritiden allgemein und stellen somit keine Qualitätsmessinstrumente speziell für RA dar [32-34]. Allen Publikationen gemeinsam ist, dass es keine einheitlich verwendeten Qualitätsmessinstrumente gibt und dass die jeweils gewählte Methodik zur Erstellung der Qualitätsmessinstrumente unterschiedlich ist.
SLR Versorgungslücken. In • Abb. 2 sind die einzelnen Schritte der SLR bezüglich der Versorgungslücken aufgeführt. Es wurden insgesamt 21 Studien für Versorgungslücken in den folgenden Domänen, identifiziert: Therapie $(n=7)$, Komorbidität ( $n=7)$, Management $(n=6)$, Risikogruppe ( $n=3)$, Überweisung $(n=3)$ und Training $(n=2)$.

Phase 2: Identifikation der

Schlüsselbereiche für die Qualitätsverbesserung

Die AG identifizierte in einem virtuellen Treffen im Oktober 2020 insgesamt 19 Schlüsselbereiche, die quantitativ erfasst werden können (- Tab. 2).

Bei der Diskussion spielten die Aspekte der Validität inklusive der Augenscheinvalidität, der Durchführbarkeit, der inhaltlichen Bedeutung des Schlüsselbereiches und der Wahrscheinlichkeit der Verwendung eine Rolle.

\section{Phase 3: Priorisierung der Versorgungslücken}

Die Priorisierung wurde in einem weiteren virtuellen Treffen im Dezember 2020 vorgestellt und intensiv diskutiert. Die AG entschied sich, insgesamt zu 8 Versorgungslücken korrespondierende QS zu formu- lieren. Die Entscheidung fußte auf einer balancierten Abwägung zwischen Versorgungslücken, die zu $100 \%$ als relevant bewertet wurden und Versorgungslücken, die einen hohen Grad der Zustimmung erhielten.

\section{Phase 4: Formulierung der} Qualitätsstandards für RA

Die Ausformulierung der QS erfolgte im Rahmen eines virtuellen Meetings, an dem 13 Mitglieder der AG teilnahmen. Die finale Version der QS findet sich in den - Tab. 3, 4, 5, 6, 7, 8, 9 und 10 und die ausführliche Besprechung der inhaltlichen Diskussion weiter unten im Text. An der Abstimmung nahmen am Ende des Treffens alle 11 noch anwesenden Mitglieder der Kommission teil.

\section{Phase 5: Konsentierung der Qualitätsstandards}

An der Abstimmung der QS nahmen insgesamt 13 AG-Mitglieder teil. Der niedrigste Grad der Zustimmung fand sich bei QS 6 (Screening psychosozialer Folgeprobleme) mit 8,7 $\pm 1,9$ und der höchste Grad der Zustimmung bei QS 4 (Konsequente The-

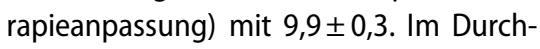
schnitt lag der Grad der Zustimmung aller QS bei 9,3 $\pm 0,7$. Die Abstimmungsergebnisse der QS sind in • Tab. 11 zu finden.

\section{Qualitätsstandards für die Versorgung von Patient*innen mit rheumatoider Arthritis}

\section{QS 1: Frühzeitige Diagnose (ब Tab. 3)}

Bei der Formulierung dieses QS wurde das Hauptaugenmerk auf eine zeitnahe Diagnosestellung gelegt, da sich alle AGMitglieder darin einig waren, dass jede Woche zählt, um das Ziel der Remission so früh wie möglich zu erreichen. Grundsätzlich wurde angemerkt, dass es bereits initial zu Verzögerungen kommen kann, da der Beginn von Diagnostik und Therapie vom Zeitpunkt der Erstvorstellung des $^{*}$ r Patient ${ }^{*}$ in bei einem ${ }^{*}$ Ärzt ${ }^{*}$ in, in der Regel bei einem* ${ }^{*}$ internistisch-rheumatologischen Facharzt*ärztin abhängig ist. Diese Vorstellung hängt unter anderem erheblich vom Leidensdruck des*r Patient*in bzw. seinem*ihrem Interesse ab, die Beschwerden zügig abzuklären. Insofern darf es in den weiteren Schritten (hausärztliche 


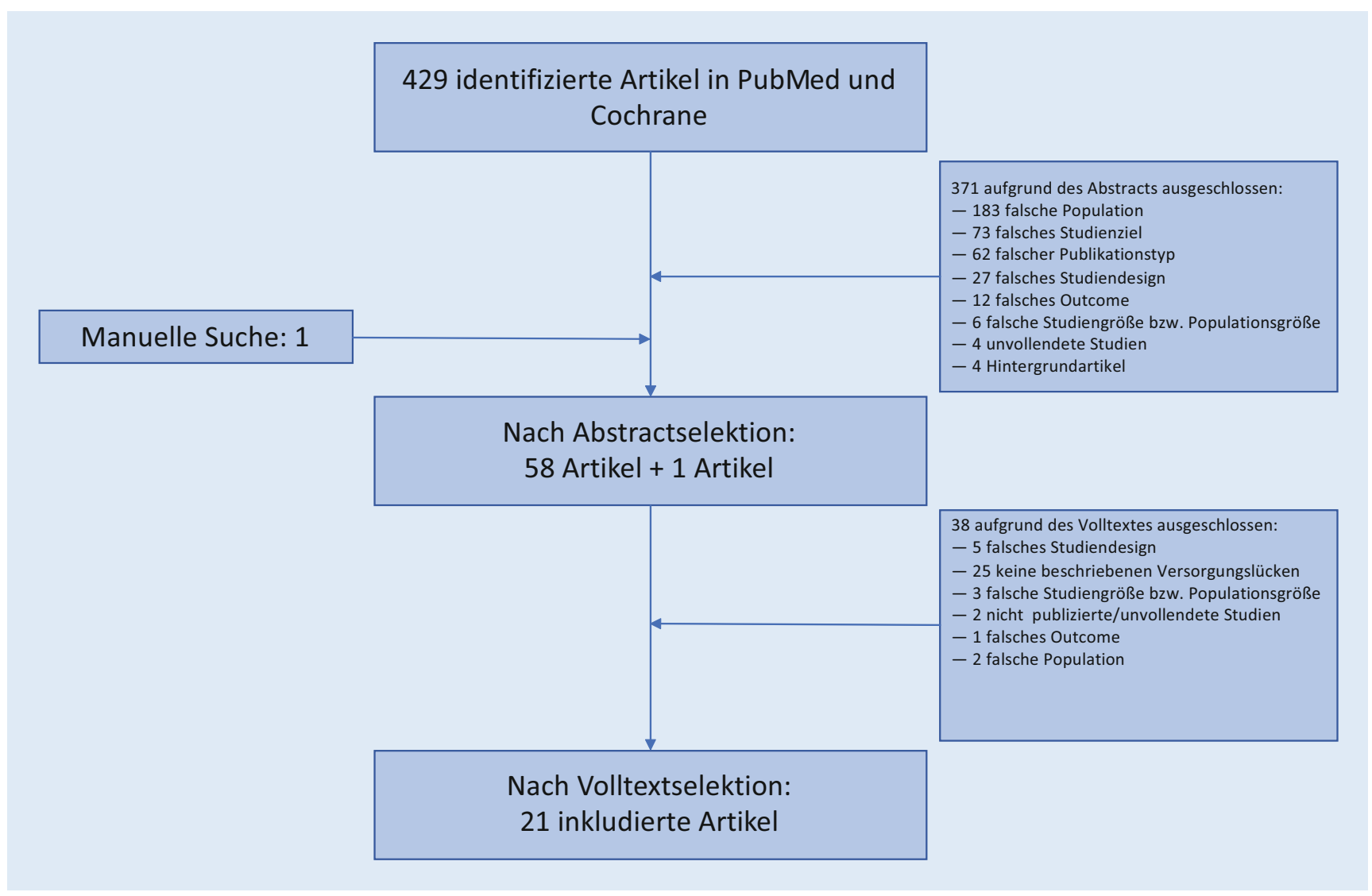

Abb. 2 ム Flussdiagramm der SLR der Phase 1b: Identifikation von relevanten Versorgungslücken

Überweisung, rheumatologische Erstvorstellung) keine weiteren Verzögerungen geben. Die Expertise eines* ${ }^{*}$ internistischrheumatologischen Facharzt*ärztin ist sowohl für die Diagnosestellung als auch für die Therapieeinleitung in den meisten Fällen erforderlich, um unnötige und die Patient*innen potenziell schädigende Folgen zu vermeiden. Deshalb werden in den meisten rheumatologischen Einrichtungen Frühsprechstunden angeboten. In manchen Fällen ist auch die Einweisung in ein rheumatologisches Akutkrankenhaus erforderlich.

Nach intensiven inhaltlichen Diskussionen stimmten alle 11 anwesenden Mitglieder für diesen $\mathrm{QS}$.

\section{QS 2: Ziel Remission (• Tab. 4)}

Zum einen wurde hier diskutiert und festgestellt, dass eine komplette Remission nicht bei allen RA-Patient*innen zu erreichen ist. Dieser Ansicht waren zwar fast alle AG-Mitglieder, jedoch wurde auch kritisch angemerkt, dass eine Teilremission kein Qualitätsmerkmal darstellen kann. In- sofern wurde entschieden, dass zwar das primäre Ziel die komplette Remission ist, jedoch davon auszugehen ist, dass diese Qualität nicht bei allen RA-Patient*innen zu erreichen ist. In der internationalen Literatur wird dieses durchaus bekannte Problem meist so gelöst, dass von einer möglichen Akzeptanz niedriger Krankheitsaktivität gesprochen wird [35, 36].

Zum anderen wurden die zur Verwendung kommenden validierten Scores zur Bestimmung der Krankheitsaktivität diskutiert. Hier wurde kritisch angemerkt, dass der DAS28 durch die darin nicht enthaltene Beurteilung der Vorfüße den Gelenkstatus nur unzureichend abbildet [37]. Zudem wurde kritisch diskutiert, dass das Nichterreichen einer Remission möglicherweise durch eine hohes Patientenglobalurteil verursacht sein könnte, wobei dadurch dann die Selbstbeurteilung im Missverhältnis zur Gelenkuntersuchung steht [38].

Die aktuellen S2e- sowie S3-LL der DGRh $[2,3]$ empfehlen zur Bestimmung der Krankheitsaktivität den CDAI, SDAI und/oder den DAS28. Basierend auf die- sen Empfehlungen wurde entschieden, dass alle 3 Scores zur Bestimmung der Krankheitsaktivität verwendet werden können.

Nach intensiven inhaltlichen Diskussionen stimmten alle 11 anwesenden Mitglieder für diesen QS.

\section{QS 3: Glukokortikoidfreiheit (• Tab. 5)}

Generell bestand Einigkeit, dass eine möglichst niedrige Glukokortikoiddosis bei RAPatient*innen anzustreben ist und dass das Ziel bei Patient*innen in Remission die Glukokortikoidfreiheit ist. Über die Höhe der Glukokortikoiddosis gab es allerdings eine intensive Diskussion zwischen den AG-Mitgliedern, da die nationalen und internationalen Leitlinien Dosierungen zwischen $<5$ und $<7,5 \mathrm{mg}$ Prednisolonäquivalent angeben [2, 3, 39]. Die AG-Mitglieder einigten sich auf die Formulierung, einen zeitlich begrenzten Dosisbereich von $\leq 7,5 \mathrm{mg}$ Prednisolonäquivalent/Tag zuakzeptieren, und präzisierten, dass bei Patient*innen, bei denen das vollständige Absetzen der Glukokortikoide nicht erreichbar ist, eine 


\begin{tabular}{|c|c|c|c|c|}
\hline \multirow[t]{2}{*}{$\begin{array}{l}\text { QS } \\
\mathrm{Nr}\end{array}$} & \multirow[t]{2}{*}{ Text QS } & \multirow{2}{*}{$\begin{array}{l}\text { Stimmen Sie zu, dass } \\
\text { diese Domäne eine rele- } \\
\text { vante Versorgungslücke } \\
\text { in Deutschland darstellt? }\end{array}$} & \multicolumn{2}{|c|}{$\begin{array}{l}\text { Bitte geben Sie den Grad der Zustim- } \\
\text { mung für diese Domäne an }\end{array}$} \\
\hline & & & Mean & SD \\
\hline 1 & Überweisung zu dem*der Rheumatolog*in & $84,6 \% \mathrm{Ja}$ & 7,8 & 2,3 \\
\hline 2 & $\begin{array}{l}\text { Frühzeitige Diagnose (innerhalb von } 6 \text { Wochen nach Vorstellung } \\
\text { bei dem*der Hausarzt*ärztin) }\end{array}$ & $92,3 \% \mathrm{Ja}$ & 9,4 & 0,7 \\
\hline 3 & Versorgung mit einer Basistherapie bei gesicherter Diagnose & $69,2 \% \mathrm{Ja}$ & 6,3 & 3,0 \\
\hline $3 a$ & $\begin{array}{l}\text { Versorgung mit einer Basistherapie bei gesicherter Diagnose: Ge- } \\
\text { samtpopulation }\end{array}$ & $61,5 \% \mathrm{Ja}$ & 6,1 & 3,2 \\
\hline $3 b$ & $\begin{array}{l}\text { Versorgung mit einer Basistherapie bei gesicherter Diagnose: Po- } \\
\text { pulationen mit Besonderheiten in der Behandlung }\end{array}$ & $84,6 \% \mathrm{Ja}$ & 7,3 & 2,8 \\
\hline 4 & $\begin{array}{l}\text { Anzahl der Patient*innen ohne Glukokortikoide nach Erreichen } \\
\text { einer Remission }\end{array}$ & $100 \% \mathrm{Ja}$ & 7,1 & 2,3 \\
\hline 5 & Interkollegialer und intersektoraler Austausch & $92,3 \% \mathrm{Ja}$ & 5,4 & 2,3 \\
\hline 6 & Interdisziplinäre Versorgung & $92,3 \% \mathrm{Ja}$ & 5,5 & 2,4 \\
\hline 7 & $\begin{array}{l}\text { Rehabilitation bei Patient*innen mit eingeschränkter Teilhabe } \\
\text { verordnen }\end{array}$ & $92,3 \% \mathrm{Ja}$ & 5,8 & 2,9 \\
\hline 8 & $\begin{array}{l}\text { Verordnung von Heil- und Hilfsmitteln bei Patient*innen mit Ein- } \\
\text { schränkung der Funktionsfähigkeit }\end{array}$ & $92,3 \% \mathrm{Ja}$ & 7,0 & 2,3 \\
\hline 9 & Selbstmanagement der Patient*innen & $84,6 \% \mathrm{Ja}$ & 4,6 & 2,7 \\
\hline $9 a$ & $\begin{array}{l}\text { Selbstmanagement der Patient*innen: Strukturierte Pa- } \\
\text { tient*innenschulung bei jedem }{ }^{*} \text { r Patient }{ }^{*} \text { in }\end{array}$ & $92,3 \% \mathrm{Ja}$ & 6,8 & 2,9 \\
\hline $9 b$ & $\begin{array}{l}\text { Selbstmanagement der Patient*innen: Jede*r Patient*in soll über } \\
\text { den Zugang zur Selbsthilfeorganisation informiert werden }\end{array}$ & $53,8 \% \mathrm{Ja}$ & 3,4 & 2,9 \\
\hline 10 & $\begin{array}{l}\text { Prävention sowie Diagnostik und Therapie einer begleitenden } \\
\text { Osteoporose bei RA }\end{array}$ & $84,6 \% \mathrm{Ja}$ & 5,8 & 2,7 \\
\hline 11 & Regelmäßige Erhebung der Krankheitsaktivität & $53,8 \% \mathrm{Ja}$ & 4,7 & 3,0 \\
\hline 12 & $\begin{array}{l}\text { Differenzialdiagnose der Schmerzen und Zugang zum Schmerzma- } \\
\text { nagement }\end{array}$ & $76,9 \% \mathrm{Ja}$ & 6,5 & 3,1 \\
\hline 13 & Notfallmanagement/kurzfristiger Zugang/schneller Termin & $92,3 \% \mathrm{Ja}$ & 7,9 & 2,4 \\
\hline 14 & $\begin{array}{l}\text { Konsequente Therapieeskalation bei Nichterreichen einer Remissi- } \\
\text { on }\end{array}$ & $100 \% \mathrm{Ja}$ & 7,2 & 2,0 \\
\hline $14 a$ & $\begin{array}{l}\text { Konsequente Therapieeskalation bei Nichterreichen einer Remissi- } \\
\text { on: medikamentöse Eskalation oder Wechsel }\end{array}$ & $100 \% \mathrm{Ja}$ & 6,9 & 2,0 \\
\hline $14 \mathrm{~b}$ & $\begin{array}{l}\text { Konsequente Therapieeskalation bei Nichterreichen einer Remissi- } \\
\text { on: Durchführung von Punktionen/Injektionen }\end{array}$ & $92,3 \% \mathrm{Ja}$ & 6,8 & 2,3 \\
\hline 15 & Screening psychosozialer Folgeprobleme & $100 \% \mathrm{Ja}$ & 6,6 & 1,7 \\
\hline 16 & Jährlicher Review & $69,2 \% \mathrm{Ja}$ & 5,6 & 3,2 \\
\hline 17 & Kontrolle Impfstatus und Ergänzung der Impflücken & $92,3 \% \mathrm{Ja}$ & 6,8 & 2,4 \\
\hline 18 & Behandlungsplan zwischen Ärzt*in und Patient*in & $69,2 \% \mathrm{Ja}$ & 3,8 & 3,6 \\
\hline 19 & $\begin{array}{l}\text { Angebote effizienter, digitaler Medien als Ergänzung zur ärztlichen } \\
\text { Versorgung }\end{array}$ & $61,5 \% \mathrm{Ja}$ & 4,9 & 3,6 \\
\hline
\end{tabular}

Dosis von maximal $5 \mathrm{mg}$ Prednisolonäquivalent/Tag als oberste akzeptable Grenze definiert wird.

Nach intensiven inhaltlichen Diskussionen stimmten alle 11 anwesenden Mitglieder für diesen QS.
QS 4: Konsequente Therapieanpassung (• Tab. 6)

Das wichtige Therapieziel Remission wird aus verschiedenen Gründen relativ häufig nicht erreicht $([36,40,41]$, s. auch QS 2). Das Hauptaugenmerkin der Diskussion bei diesem QS lag in der möglicherweise fehlenden, aber zum Teil durchaus notwendigen Ursachenforschung für die nicht erreichte Remission durch den*die Rheumatolog*in.
Dabei wurde betont, dass das frühzeitige Erkennen von begleitenden schmerzbegünstigenden Erkrankungen wie Arthrose und Fibromyalgie differenzialdiagnostisch von großer Bedeutung ist. Daher wurde in diesem QS nicht nur die konsequente Therapieanpassung bei nicht erreichter Remission, sondern auch „[...] die differenzialdiagnostische Aufarbeitung der Ur- 
Tab. 3 Qualitätsstandard 1: Frühzeitige Diagnose

\begin{tabular}{|c|c|c|c|c|c|}
\hline Domäne & Standard & Rationale & $\begin{array}{l}\text { Qualitätsmessung, Katego- } \\
\text { rie Struktur }\end{array}$ & $\begin{array}{l}\text { Qualitäts- } \\
\text { messung, } \\
\text { Kategorie } \\
\text { Prozess, }\end{array}$ & $\begin{array}{l}\text { Qualitäts- } \\
\text { messung, } \\
\text { Kategorie } \\
\text { Prozess, }\end{array}$ \\
\hline $\begin{array}{l}\text { Frühzeitige } \\
\text { Diagnose }\end{array}$ & $\begin{array}{l}\text { Bei Pa- } \\
\text { tient*innen } \\
\text { mit neu } \\
\text { aufge- } \\
\text { tretenen } \\
\text { Gelenk- } \\
\text { schmerzen } \\
\text { und Ver- } \\
\text { dacht auf } \\
\text { RA wird die } \\
\text { Diagnose } \\
\text { inner- } \\
\text { halb von } \\
6 \text { Wochen } \\
\text { gesichert }\end{array}$ & $\begin{array}{l}\text { Die Symptome der RA, insbesondere die frühen, zum } \\
\text { Teil unspezifischen Symptome, werden von Nicht- } \\
\text { Rheumatolog*innen oft nicht als Manifestation einer } \\
\text { entzündlich-rheumatischen Erkrankung erkannt. Da- } \\
\text { durch unterbleibt die Durchführung diagnostischer } \\
\text { Maßnahmen, und es kommt zu einer erheblichen Ver- } \\
\text { zögerung bei Diagnose und Therapieeinleitung. Ra- } \\
\text { diologisch nachweisbare Gelenkdestruktionen und } \\
\text { Funktionseinschränkungen entwickeln sich gerade zu } \\
\text { Beginn der Erkrankung am stärksten. Das Therapie- } \\
\text { ziel für Patient*innen mit RA ist die Remission (s. QS 2). } \\
\text { Das Erreichen einer Remission ist umso wahrscheinli- } \\
\text { cher, je früher die Diagnose einer RA gestellt und eine } \\
\text { Therapie eingeleitet wird. Die Anzahl aufgetretener } \\
\text { Erosionen korreliert mit der zeitlichen Verzögerung bis } \\
\text { zur Einleitung der ersten Therapie. Wenn der Verdacht } \\
\text { auf RA besteht, geben die EULAR-Empfehlungen der } \\
\text { frühen RA, die ACR-Klassifikationskriterien und die S3- } \\
\text { Leitlinie für frühe RA der DGRh eine Orientierung für } \\
\text { die Diagnosefindung. Die Patient*innen, die sich mit } \\
\text { Symptomen einer RA ärztlich vorstellen, sollen zügig } \\
\text { in die Rheumatologie überweisen werden. Da es ei- } \\
\text { ne Vielzahl an Differenzialdiagnosen abzuklären gibt, } \\
\text { ist die Fachexpertise der Rheumatologie erforderlich. } \\
\text { Frühsprechstunden, in denen Patient*innen mit noch } \\
\text { unklaren Arthralgien frühzeitig auf das mögliche Vorlie- } \\
\text { gen einer RA untersucht werden, können wesentlich zur } \\
\text { rechtzeitigen Diagnosestellung beitragen }\end{array}$ & $\begin{array}{l}\text { Vorhaltung von Maßnahmen } \\
\text { und Strukturen, die eine frü- } \\
\text { he Diagnose ermöglichen. } \\
\text { Dies schließt nicht nur eine } \\
\text { ausreichende Anzahl von } \\
\text { Rheumatolog*innen und ent- } \\
\text { sprechende Kapazitäten in } \\
\text { Frühsprechstunden ein, son- } \\
\text { dern auch Maßnahmen zur } \\
\text { Fortbildung der primärärztli- } \\
\text { chen Versorgungsebene und } \\
\text { anderer Gesundheitsdienst- } \\
\text { leister, z. B. durch rheumato- } \\
\text { logische Zentren. Dazu zählen } \\
\text { aber auch Informationsange- } \\
\text { bote für die Bevölkerung- } \\
\text { auch durch die Deutsche } \\
\text { Rheumaliga -, um die Sen- } \\
\text { sibilisierung für Anzeichen } \\
\text { und Frühsymptome einer } \\
\text { RA zu erhöhen und zu för- } \\
\text { dern, damit Patient*innen mit } \\
\text { Verdacht auf RA innerhalb } \\
\text { von } 6 \text { Wochen diagnostiziert } \\
\text { werden können }\end{array}$ & $\begin{array}{l}\text { Zähler } \\
\text { neudiagno- } \\
\text { stizierten } \\
\text { RA-Pa- } \\
\text { tient*innen, } \\
\text { bei denen } \\
\text { die Dia- } \\
\text { gnose } \\
\text { innerhalb } \\
\text { von } 6 \text { Wo- } \\
\text { chen nach } \\
\text { Symptom- } \\
\text { beginn } \\
\text { fachärztlich } \\
\text { gestellt } \\
\text { wurde }\end{array}$ & $\begin{array}{l}\text { Avnener } \\
\text { Alle Ra- } \\
\text { tient*innen, } \\
\text { bei denen } \\
\text { im letzten } \\
\text { Jahr eine RA } \\
\text { fachärztlich } \\
\text { neu dia- } \\
\text { gnostiziert } \\
\text { wurde }\end{array}$ \\
\hline
\end{tabular}

sache(n) [...]" als klares Ziel für diesen QS formuliert.

Nach intensiven inhaltlichen Diskussionen stimmten alle 11 anwesenden Mitglieder für diesen $\mathrm{QS}$.

\section{QS 5: Konsequente Therapie der eingeschränkten Funktionsfähigkeit (- Tab. 7)}

Bei diesem QS wurden verschiedene Aspekte diskutiert. Grundsätzlich bestehen bei Patient*innen mit RA häufig Komorbiditäten, die zu entsprechenden Funktionseinschränkungen führen können. Ein wichtiges Ziel des*r Rheumato$\log ^{*}$ in ist daher die Differenzierung solcher Ursachen von Funktionseinschränkungen von der durch die RA bedingten eingeschränkten Funktionsfähigkeit. Hierbei spielt auch die Unterscheidung zwischen einer akut eingeschränkten Funktionsfähigkeit durch aktive Entzündung und die durch einen bereits eingetretenen strukturellen Schaden, wie z. B. durch sekundäre Arthrose, eine Rolle.
Darüber hinaus wurde kritisch angemerkt, dass es keine validierten Scores gibt, die einen Schwellenwert für eingeschränkte Funktionsfähigkeit festlegen. Die nun in dem QS verwendeten Schwellenwerte bezüglich eingeschränkter Funktionsfähigkeit basieren aufExpert*innenmeinung sowie auf Auswertungen der Kerndokumentation [42]. In diesem Zusammenhang wird aber auf die grundsätzliche Limitation solcher Schwellenwerte verwiesen, da diese u. a. nicht das Alter, den BMI, (Fehlen) körperlicher Aktivität und den Lebensstil und den damit einhergehenden Funktionsverlust berücksichtigen [43].

Außerdem gibt es erhebliche individuelle Unterschiede bei Patient*innen mit RA in der Krankheitsverarbeitung, was z. B. das Coping mit der Krankheit mit einschließt und dass zum anderen auch die Umgebungssituation eine nicht geringe Rolle spielen kann - hierbei geht es um Verwandte, Freunde und die möglicherweise nicht behindertengerechte Wohnsituation oder auch die Verhältnisse am Arbeitsplatz.
Nach intensiven inhaltlichen Diskussionen stimmten alle 11 anwesenden Mitglieder für diesen QS.

\section{QS 6: Screening psychosozialer Folgeprobleme (0 Tab. 8)}

Psychosoziale Folgeprobleme können für Patient*innen und Rheumatolog*innen mit RA in der Kommunikation ein schwierig anzusprechendes Thema sein, daher war den meisten AG-Mitgliedern ein gesonderter QS besonders wichtig. Hierbei stand nicht nur das Erkennen von entsprechenden Problemen (s. auch QS 5) im Vordergrund, sondern auch, ggf. in Kooperation mit anderen Fachabteilungen, das Einleiten von Maßnahmen, um Probleme ggf. zu beheben oder gegebene Einschränkungen zu verbessern. In der Diskussion dieses QS wurde daher ein großes Augenmerk auf die interdisziplinäre Zusammenarbeit bei der Lösung psychosozialer Probleme gelegt. Die psychische Komponente spielt nicht nur in der Lebensqualität der Patient*innen 
Tab. 4 Qualitätsstandard 2: Ziel Remission

\begin{tabular}{|c|c|c|c|c|c|}
\hline Domäne & Standard & Rationale & $\begin{array}{l}\text { Qualitätsmessung, Katego- } \\
\text { rie Struktur }\end{array}$ & $\begin{array}{l}\text { Qualitäts- } \\
\text { messung, } \\
\text { Kategorie } \\
\text { Prozess, } \\
\text { Zähler }\end{array}$ & $\begin{array}{l}\text { Qualitäts- } \\
\text { messung, } \\
\text { Kategorie } \\
\text { Prozess, } \\
\text { Nenner }\end{array}$ \\
\hline $\begin{array}{l}\text { Ziel Remissi- } \\
\text { on }\end{array}$ & $\begin{array}{l}\text { Das Ziel } \\
\text { der Be- } \\
\text { handlung } \\
\text { von Pa- } \\
\text { tient*innen } \\
\text { mit RA ist } \\
\text { die Re- } \\
\text { mission }\end{array}$ & $\begin{array}{l}\text { Eine erhöhte Krankheitsaktivität bei RA ist mit erhöhter } \\
\text { Mortalität, zunehmenden Gelenkdestruktionen und } \\
\text { Funktionseinschränkung sowie entsprechenden so- } \\
\text { zioökonomischen Konsequenzen assoziiert. Mit dem } \\
\text { raschen Erreichen einer Remission werden nicht nur } \\
\text { krankheitsspezifische Komplikationen vermieden, son- } \\
\text { dern es wird auch die Wahrscheinlichkeit erhöht, dass } \\
\text { die Remission über einen längeren Zeitraum anhält. } \\
\text { Die S3-Leitlinie zur Diagnose einer frühen RA stellt fest, } \\
\text { dass eine Remission nach } 3 \text { (spätestens 6) Monaten er- } \\
\text { reicht werden soll. Je früher die Remission erreicht wird, } \\
\text { desto wahrscheinlicher hält diese auch länger an - und } \\
\text { dies z.T. sogar mit reduzierter Dosis verordneter Me- } \\
\text { dikamente. Die Krankheitsaktivität von Patient*innen } \\
\text { mit RA sollte mit validierten Scores bewertet werden. } \\
\text { Entsprechend der S3-Leitlinie zur Diagnose einer frü- } \\
\text { hen RA, der S2e-Leitlinie zur Therapie einer RA der } \\
\text { DGRh sowie den EULAR-Empfehlungen zum Manage- } \\
\text { ment der frühen RA wird empfohlen, den „simplified } \\
\text { disease activity score“ (SDAI), den „clinical disease ac- } \\
\text { tivity index“ (CDAI) oder den „disease activity score“ } \\
\text { (DAS28) zu verwenden. Demnach ist Krankheitsremissi- } \\
\text { on als SDAI< 3,3, CDAI<2,8 oder - weniger spezifisch - } \\
\text { DAS28 <2,6 definiert. Das Ziel ist grundsätzlich nicht } \\
\text { nur das Erreichen, sondern auch das Aufrechterhalten } \\
\text { der erreichten Remission. Bei Nicht-Erreichen einer Re- } \\
\text { mission, sollen alle Möglichkeiten einer intersektoralen } \\
\text { Versorgung ausgeschöpft werden und weitere medika- } \\
\text { mentöse sowie nichtmedikamentöse Therapieverfahren } \\
\text { zur Anwendung kommen. Dabei ist die konsequente } \\
\text { Intensivierung der Therapie bei Nichterreichen einer } \\
\text { Remission ein wesentlicher Baustein des Therapiekon- } \\
\text { zeptes (s. QS 4) }\end{array}$ & $\begin{array}{l}\text { Vorhaltung von Maßnahmen } \\
\text { und Strukturen, einschließlich } \\
\text { einer ausreichenden Anzahl } \\
\text { von Rheumatolog*innen, mit } \\
\text { uneingeschränktem Zugang } \\
\text { (z. B. keine Fallzahldecke- } \\
\text { lung) sowie Gewährleistung } \\
\text { einer intersektoralen The- } \\
\text { rapie, um sicherzustellen, } \\
\text { dass Patient*innen mit RA } \\
\text { regelmäßig mit validierten } \\
\text { Messinstrumenten evaluiert } \\
\text { werden und die Therapie ge- } \\
\text { mäß der S3-Leitlinie sowie } \\
\text { der S2e-Leitlinie mit dem } \\
\text { Ziel der Remission angepasst } \\
\text { werden kann }\end{array}$ & $\begin{array}{l}\text { RA-Pa- } \\
\text { tient*innen } \\
\text { in Remissi- } \\
\text { on }\end{array}$ & $\begin{array}{l}\text { Alle Pa- } \\
\text { tient*innen } \\
\text { mit RA }\end{array}$ \\
\hline
\end{tabular}

Tab. 5 Qualitätsstandard 3: Glukokortikoidfreiheit

\begin{tabular}{|c|c|c|c|c|c|}
\hline Domäne & Standard & Rationale & $\begin{array}{l}\text { Qualitätsmessung, Kategorie } \\
\text { Struktur }\end{array}$ & $\begin{array}{l}\text { Qualitäts- } \\
\text { messung, } \\
\text { Kategorie } \\
\text { Prozess, } \\
\text { Zähler }\end{array}$ & $\begin{array}{l}\text { Qualitäts- } \\
\text { messung, } \\
\text { Kategorie } \\
\text { Prozess, } \\
\text { Nenner }\end{array}$ \\
\hline $\begin{array}{l}\text { Glukokorti- } \\
\text { koidfreiheit }\end{array}$ & $\begin{array}{l}\text { Bei Pa- } \\
\text { tient*in- } \\
\text { nen mit } \\
\text { RA in Re- } \\
\text { mission } \\
\text { ist Gluko- } \\
\text { kortiko- } \\
\text { idfreiheit } \\
\text { das Ziel }\end{array}$ & $\begin{array}{l}\text { Eine dauerhafte Glukokortikoidtherapie führt zu einer } \\
\text { Vielzahl unerwünschter Wirkungen. Hierzu zählen unter } \\
\text { anderem kardiovaskuläre Ereignisse, erhöhte Infektan- } \\
\text { fälligkeit, mit Frakturen einhergehende Osteoporose, } \\
\text { gastrointestinale Komplikationen und auch selten eine } \\
\text { sekundäre Nebennierenrindeninsuffizienz. Die gluko- } \\
\text { kortikoidfreie Remission ist daher das primäre Ziel der } \\
\text { Therapie. Gemäß der S3-Leitlinie zur Diagnose einer frü- } \\
\text { hen RA, der S2e-Leitlinie zur Therapie der RA sowie den } \\
\text { EULAR-Empfehlungen zum Management einer (frühen) } \\
\text { RA der DGRh wird bis zum Erreichen der Wirkung einer } \\
\text { csDMARD-Therapie die Krankheitsaktivität mit einer Glu- } \\
\text { kokortikoidtherapie unterdrückt. Die Glukokortikoiddosis } \\
\text { soll dann aber innerhalb von } 8 \text { Wochen in den Low-Dose- } \\
\text { Bereich ( } \leq 7,5 \text { mg/Tag Prednisolonäquivalent) reduziert } \\
\text { und nach } 3 \text { bis } 6 \text { Monaten nach Möglichkeit beendet } \\
\text { werden. Bei Patient*innen, bei denen das vollständige } \\
\text { Absetzen der Glukokortikoide nicht erreichbar ist, wird } \\
\text { eine Dosis von maximal } 5 \text { mg Prednisolonäquivalent/Tag } \\
\text { als oberste akzeptable Grenze definiert }\end{array}$ & $\begin{array}{l}\text { Vorhaltung von Maßnahmen } \\
\text { und Strukturen, einschließlich } \\
\text { des Zugangs zu allen medika- } \\
\text { mentösen Therapiemöglich- } \\
\text { keiten, um die Chance auf eine } \\
\text { glukokortikoidfreie Remission } \\
\text { zu maximieren }\end{array}$ & $\begin{array}{l}\text { RA-Pa- } \\
\text { tient*innen } \\
\text { in Remis- } \\
\text { sion ohne } \\
\text { Glukokorti- } \\
\text { koide }\end{array}$ & $\begin{array}{l}\text { Alle } \\
\text { RA-Pa- } \\
\text { tient*in- } \\
\text { nen in } \\
\text { Remission }\end{array}$ \\
\hline
\end{tabular}


Tab. 6 Qualitätsstandard 4: Konsequente Therapieanpassung

\begin{tabular}{|c|c|c|c|c|c|}
\hline Domäne & Standard & Rationale & $\begin{array}{l}\text { Qualitätsmessung, Kategorie } \\
\text { Struktur }\end{array}$ & $\begin{array}{l}\text { Qualitäts- } \\
\text { messung, } \\
\text { Kategorie } \\
\text { Prozess, } \\
\text { Zähler }\end{array}$ & $\begin{array}{l}\text { Qualitäts- } \\
\text { messung, } \\
\text { Kategorie } \\
\text { Prozess, } \\
\text { Nenner }\end{array}$ \\
\hline $\begin{array}{l}\text { Konsequente } \\
\text { Therapie- } \\
\text { anpassung }\end{array}$ & $\begin{array}{l}\text { Bei Nicht- } \\
\text { erreichen } \\
\text { der Re- } \\
\text { mission } \\
\text { gilt es, die } \\
\text { Ursachen } \\
\text { differen- } \\
\text { zialdia- } \\
\text { gnostisch } \\
\text { aufzuar- } \\
\text { beiten } \\
\text { und die } \\
\text { Therapie } \\
\text { entspre- } \\
\text { chend } \\
\text { zu ad- } \\
\text { aptieren }\end{array}$ & $\begin{array}{l}\text { Eine nicht unbedeutende Zahl von Patient*innen gilt als } \\
\text { therapierefraktär oder schwer behandelbar, trotz kor- } \\
\text { rekter Diagnosestellung und regelmäßiger Evaluation } \\
\text { der Krankheitsaktivität. Für die Versorgung dieser Pa- } \\
\text { tient*innen bieten die aktuellen EULAR-Empfehlungen } \\
\text { für die „Difficult-to-treat“-RA sowie die S2e-Leitlinie zur } \\
\text { Therapie einer RA der DRGh evidenzbasierte Informatio- } \\
\text { nen. Dabei gilt es, differenzialdiagnostische Überlegun- } \\
\text { gen hinsichtlich der Ursache des Nichterreichens oder } \\
\text { des sekundären Verlustes der Remission anzustellen. Da } \\
\text { die Unterscheidung zwischen bestehender entzündlich } \\
\text { bedingter Krankheitsaktivität und anderen Ursachen wie } \\
\text { sekundären Schmerzerkrankungen und Arthrose zum } \\
\text { Teil schwierig zu differenzieren ist, bedarf es der Fach- } \\
\text { expertise von Rheumatolog*innen. Bei Nichterreichen } \\
\text { oder einem sekundären Verlust einer Remission, sol- } \\
\text { len alle Möglichkeiten einer intersektoralen Versorgung } \\
\text { ausgeschöpft werden und medikamentöse sowie nicht- } \\
\text { medikamentöse Therapieverfahren, wie z. B. Kälte- oder } \\
\text { Wärmetherapie, Physiotherapie und/oder Ergotherapie } \\
\text { zur Anwendung kommen. Die medikamentöse Therapie } \\
\text { muss überprüft und ggf. umgestellt werden. Darüber } \\
\text { hinaus können interventionelle Verfahren wie eine intra- } \\
\text { artikuläre Glukokortikoidinjektion erforderlich sein. Die } \\
\text { Entwicklung von Komorbiditäten ist bei der Therapieent- } \\
\text { scheidung mit zu berücksichtigen }\end{array}$ & $\begin{array}{l}\text { Vorhaltung von Maßnahmen } \\
\text { und Strukturen einschließlich } \\
\text { einer ausreichenden Anzahl } \\
\text { von Rheumatolog*innen } \\
\text { (Facharztstandard), um bei } \\
\text { Nichterreichen einer Remis- } \\
\text { sion gezielte differenzialdi- } \\
\text { agnostische Überlegungen } \\
\text { anzustellen und einen un- } \\
\text { eingeschränkten Zugang zu } \\
\text { allen Therapiemöglichkeiten } \\
\text { zu ermöglichen, um notwen- } \\
\text { dige Therapieadaptationen } \\
\text { durchführen zu können }\end{array}$ & $\begin{array}{l}\text { RA-Pa- } \\
\text { tient*innen, } \\
\text { bei de- } \\
\text { nen eine } \\
\text { Therapiead- } \\
\text { aptation bei } \\
\text { Nichterrei- } \\
\text { chen einer } \\
\text { Remission } \\
\text { vorgenom- } \\
\text { men wurde }\end{array}$ & $\begin{array}{l}\text { Alle } \\
\text { RA-Pa- } \\
\text { tient*in- }^{*} \text { nen, die } \\
\text { nicht in } \\
\text { Remission } \\
\text { sind }\end{array}$ \\
\hline
\end{tabular}

eine Rolle, sondern beeinflusst auch erheblich das Erreichen einer Remission. Verlaufsbeobachtungen zeigen, dass RAPatient*innen mit depressiven Symptomen im weiteren Verlauf deutlich seltener und langsamer eine Remission erreichen als RA-Patient*innen ohne ein solches Problem [44].

Nach intensiven inhaltlichen Diskussionen stimmten alle 11 anwesenden Mitglieder für diesen $\mathrm{QS}$.

\section{QS 7: Notfall- und Akutmanagement (- Tab. 9)}

Ein strittiges Thema war die Definition eines rheumatologischen "Notfalls". Hier bestand Einigkeit, dass es eine möglichst klare Differenzierung zwischen einem (objektiven) medizinischen Notfall und einem (subjektiven) individuellen akuten Versorgungsbedarf geben sollte. Da die Abgrenzung im Einzelfall jedoch schwierig sein kann, einigten sich die Mitglieder nach langer Diskussion dann auf diese hier gegebene Formulierung für das „Notfall- und Akutmanagement". Bei der Umsetzung sind nach Meinung der Kommissionsmitglieder dann v.a. auch Aspekte der Machbarkeit von Bedeutung.

Nach intensiven inhaltlichen Diskussionen stimmten alle 11 anwesenden Mitglieder für diesen QS.

\section{QS 8: Komorbiditätenerfassung/ Management (• Tab. 10)}

Eine Haupttodesursache von RA-Patient*innen sind nach wie vor kardiovaskuläre Ereignisse [45]. Daher wurde von einigen AG-Mitgliedern befürwortet, dass das Screening auf kardiovaskuläre Risikofaktoren - wie von der EULAR empfohlen [46] - einen eigenen QS darstellen sollte. Dementgegen gaben jedoch andere AG-Mitglieder zu bedenken, dass in der aktuellen Versorgungslandschaft in Deutschland der*die Rheumatolog*in keineswegs allein oder hauptamtlich für das Screening auf Komorbiditäten verantwortlich ist und daher hierfür nicht 2 separate QS gebildet werden müssen. Letztlich wurde sich darauf geeinigt, einen ausführlichen QS zu entwickeln, in dem alle relevanten Komorbiditäten gleichermaßen gewürdigt werden und auf die
Notwendigkeit einer aktiven Teilnahme des*der Hausarzt*ärztin sowie weiterer Fachexpert*innen hingewiesen bzw. diese auch eingefordert wird.

Nach intensiven inhaltlichen Diskussionen stimmten alle 11 anwesenden Mitglieder für diesen QS.

\section{Diskussion}

Die hier erstmals veröffentlichten QS der DGRh in Kooperation mit VRA und BDRh stellen für die rheumatologische Versorgung in Deutschland einen Meilenstein dar. Die Expertengruppe hat $8 \mathrm{QS}$ für wichtige Bereiche der Versorgung von RA-Patient*innen definiert. Zum ersten Mal gibt es damit auch eine Grundlage und Vorgaben für Qualitätsmessungen, wobei nicht nur die Struktur- und Prozessqualität, sondern auch die Ergebnisqualität eine Rolle spielen sollte (s. unten).

Die hier vorgestellten QS basieren auf den Vorarbeiten und verschiedenen anderen Initiativen der DGRh [47-50]. Hinsichtlich der Strukturqualität gibt es für internistisch-rheumatologische Akutkran- 
Tab.7 Qualitätsstandard 5: Konsequente Therapie der eingeschränkten Funktionsfähigkeit

\begin{tabular}{|c|c|c|c|c|c|}
\hline Domäne & Standard & Rationale & $\begin{array}{l}\text { Qualitätsmessung, Kategorie } \\
\text { Struktur }\end{array}$ & $\begin{array}{l}\text { Qualitäts- } \\
\text { messung, } \\
\text { Kategorie } \\
\text { Prozess, } \\
\text { Zähler }\end{array}$ & $\begin{array}{l}\text { Qualitäts- } \\
\text { messung, } \\
\text { Kategorie } \\
\text { Prozess, } \\
\text { Nenner }\end{array}$ \\
\hline $\begin{array}{l}\text { Konsequente } \\
\text { Therapie } \\
\text { der einge- } \\
\text { schränkten } \\
\text { Funktions- } \\
\text { fähigkeit }\end{array}$ & $\begin{array}{l}\text { RA-Pa- } \\
\text { tient*in- } \\
\text { nen } \\
\text { mit Ein- } \\
\text { schrän- } \\
\text { kung der } \\
\text { Funkti- } \\
\text { onsfä- } \\
\text { higkeit } \\
\text { werden } \\
\text { thera- } \\
\text { peutische } \\
\text { Maßnah- } \\
\text { men zur } \\
\text { Wieder- } \\
\text { herstel- } \\
\text { lung der } \\
\text { Funkti- } \\
\text { onsfä- } \\
\text { higkeit } \\
\text { ange- } \\
\text { boten }\end{array}$ & $\begin{array}{l}\text { Im Laufe der Erkrankung kann es infolge unzureichend } \\
\text { kontrollierter Krankheitsaktivität zu Gelenkdestruktionen } \\
\text { und Funktionseinschränkungen kommen. Es ist Aufgabe } \\
\text { des/der Rheumatolog*in, die Funktionseinschränkun- } \\
\text { gen mit validierten Messinstrumenten (FFbH und/oder } \\
\text { HAQ sowie der Neutral-Null-Methode) festzustellen und } \\
\text { zu dokumentieren. Die Schwellenwerte für eine Funkti- } \\
\text { onseinschränkung liegen laut Expertenkonsensus und } \\
\text { vorausgegangenen FFbH-Itemanalysen im Datensatz } \\
\text { der Kerndokumentation bei einem FFbH } 570 \% \text { und/ } \\
\text { oder einem HAQ von } \leq 0,5 \text {. Im Rahmen dessen muss } \\
\text { der/die Rheumatolog*in unter Berücksichtigung be- } \\
\text { stehender Komorbiditäten einschätzen, ob die festge- } \\
\text { stellte Funktionseinschränkung Folge der RA ist. Dabei } \\
\text { kommt der Unterscheidung zwischen Krankheitsaktivi- } \\
\text { tät und Strukturschaden hinsichtlich der Abschätzung } \\
\text { des Therapieerfolges eine wesentliche Bedeutung zu. } \\
\text { Bei bestimmten Patient*innen (z. B. bei Multimorbidität) } \\
\text { kann die klinische Bewertung auch ohne entsprechen- } \\
\text { de Patient*innenfragebögen ausreichen. Wurde ein } \\
\text { Funktionsdefizit dokumentiert, ist es Aufgabe der/des } \\
\text { Rheumatolog*in, entsprechende Verordnungen für Re- } \\
\text { habilitationsmaßnahmen, Heil- und Hilfsmittel sowie ggf. } \\
\text { eine akutstationäre Einweisung zur Komplextherapie } \\
\text { zu veranlassen. Darüber hinaus ist es Aufgabe des/der } \\
\text { Rheumatolog*in, die Durchführung und den Erfolg der } \\
\text { verordneten Maßnahmen zu kontrollieren und zu do- } \\
\text { kumentieren. Hierzu ist eine enge Interaktion zwischen } \\
\text { behandelnden Physio- und Ergotherapeut*innen und } \\
\text { Rheumatolog*innen erforderlich }\end{array}$ & $\begin{array}{l}\text { Vorhaltung von Maßnahmen } \\
\text { und Strukturen, einschließlich } \\
\text { einer ausreichenden Anzahl } \\
\text { von rheumatologischem Fach- } \\
\text { personal (Rheumatolog*innen } \\
\text { und rheumatologischer } \\
\text { Fachassistenz [RFA]) sowie } \\
\text { von Physio- und Ergothera- } \\
\text { peut*innen mit ausreichen- } \\
\text { den Kenntnissen hinsichtlich } \\
\text { rheumatologischer Funkti- } \\
\text { onseinschränkungen, um das } \\
\text { Auftreten von Funktionsein- } \\
\text { schränkungen regelmäßig zu } \\
\text { erfassen, zu dokumentieren } \\
\text { und zu behandeln. Neben der } \\
\text { personellen Ressource muss } \\
\text { auch eine ausreichend hohe } \\
\text { Kapazität an rheumatologisch } \\
\text { zugewiesenen Betten für die } \\
\text { akutstationäre sowie rehabili- } \\
\text { tative Versorgung vorhanden } \\
\text { sein } \\
\text { Gemäß der aktuellen Heil- } \\
\text { mittel-Richtlinie darf es bei } \\
\text { entsprechender Notwen- } \\
\text { digkeit der Therapien keine } \\
\text { Budgetierung geben }\end{array}$ & $\begin{array}{l}\text { Alle RA-Pa- } \\
\text { tient*innen } \\
\text { mit Funk- } \\
\text { tionsein- } \\
\text { schränkung, } \\
\text { denen } \\
\text { Rehabilita- } \\
\text { tionsmaß- } \\
\text { nahmen, } \\
\text { Heil- und } \\
\text { Hilfsmit- } \\
\text { tel und/ } \\
\text { oder akut- } \\
\text { stationäre } \\
\text { Komplex- } \\
\text { therapie } \\
\text { angeboten } \\
\text { wurden }\end{array}$ & $\begin{array}{l}\text { Alle } \\
\text { RA-Pa- } \\
\text { tient*in- } \\
\text { nen mit } \\
\text { Funkti- } \\
\text { onsein- } \\
\text { schrän- } \\
\text { kungen }\end{array}$ \\
\hline
\end{tabular}

kenhäuser bereits seit 10 Jahren eine klare Festlegung [24] und seit einigen Jahren auch ein vom AQUA-Institut verliehenes Gütesiegel [51], was unter anderem ein erfolgreiches Benchmarking von mehreren im VRA organisierten Kliniken beinhaltet [52]. Die hiermit verbundenen Projekte OBRA ("outcome benchmarking" in der rheumatologischen Akutversorgung) [23] und KOBRA $[22,51]$ wurden initial vom Bundesministerium für Gesundheit gefördert.

In den hier entwickelten QS geht es v. a. um Prozess-, aber auch um Ergebnisqualität. Da stehen die rechtzeitige Diagnosestellung und Therapieeinleitung im Fokus, die natürlich auch von vielen externen Faktoren wie einer rechtzeitigen Überweisung abhängen, aber auch von internen Prozess- und Strukturmerkmalen wie einem funktionierenden Angebot an Frühsprechstunden und der Verfügbarkeit einer ausreichenden Zahl an internistischen Rheumatolog*innen [49]. Vor allem beste- hen Probleme in Form einer begrenzten Anzahl an Weiterbildungsstellen, der Konzentration auf besonders attraktive Regionen und Ballungsräume wie etwa Berlin, München oder Hamburg und einer bis jetzt fehlenden staatlichen Förderung und Planung von Weiterbildungsstellen. Nichtsdestoweniger geben die vorhandenen internistisch-rheumatologischen Einrichtungen ihr Bestes, um die beträchtliche Zahl von Patient*innen mit entzündlich rheumatischen Erkrankungen adäquat zu versorgen. Als das Fach in der Medizin, in welchem bei den betroffenen Patient*innen so gut wie alle Organsysteme betroffen sein können - bei der RA ist es v.a. die Lunge [45] -, ist die internistische Rheumatologie genuin interdisziplinär aufgestellt [53], was sich entsprechend auch in aktuellen Versorgungsstrukturen wie der ambulanten spezialfachärztlichen Versorgung (ASV) konzeptionell niederschlägt [54].
Das Therapieziel Remission ist in der internationalen Rheumatologie unstrittig. Im Wesentlichen umfasst es die konsequente regelmäßig überprüfte weitgehende Abwesenheit von Krankheitsaktivität. Die gängigen Definitionen für Remissionen beinhalten nicht die vollständige oder weitestgehende Abwesenheit von Krankheitsaktivität wie die Boolean-Kriterien [55]. Dass der Prozentsatz von RA-Patient*innen, bei denen Remission erreicht werden kann, ein gutes mögliches Kriterium darstellt, zeigt z. B. auch die CAPEAStudie an, in der $40 \%$ der eingeschlossenen RA-Patient*innen eine Remission erreichten [56]. Durch frühzeitige Diagnose und Therapie und stringente Befolgung des T2T-Prinzips wie im rheinland-pfälzischen Rheumanetzwerk ADAPTHERA [57] lassen sich deutlich höhere Werte erzielen.

Glukokortikoide sind klinisch gut und schnell wirksam, haben in hohen Dosierungen über lange Zeiträume aber gut bekannte Nebenwirkungen wie Osteoporo- 


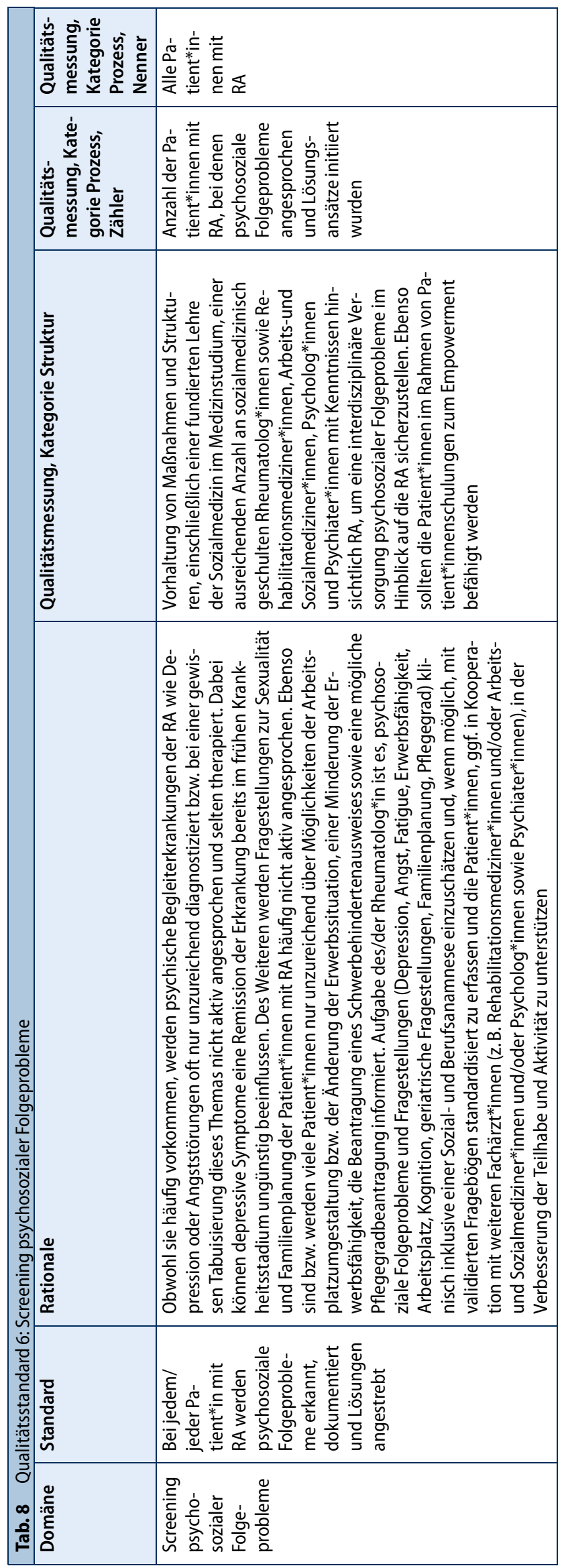

se, Diabetes und Infektionen [39, 58]. Deshalb empfiehlt die EULAR eine wesentliche Reduktion bzw. das Absetzen von Glukokortikoiden innerhalb von 6 Monaten [39]. Dies wird aber bekanntermaßen von vielen Patient*innen nicht erreicht [56]. Auf der anderen Seite sind auch geringe Dosierungen $<5 \mathrm{mg}$ Prednisolonäquivalent/Tag klinisch und sogar hinsichtlich der Röntgenprogression wirksam [59]. Deshalb geht es dabei v.a. um den Versuch der Reduktion bzw. des Absetzens, denn das unterscheidet sich erheblich von der Strategie, initial mit Glukokortikoiden zu beginnen und dann dabei einfach nur pragmatisch zu bleiben.

Die konsequente Therapieanpassung ist eng mit den QS 2 und 3 verbunden, und ein sehr wichtiger Aspekt ist auch dabei das T2T-Prinzip im Sinne der konsequenten Eskalation der Medikation, wenn die Remission noch nicht erreicht ist, aber erreicht werden kann. Dabei geht es allerdings aber nicht nur darum, sondern letztlich um jede Form der Therapieanpassung im Sinne eines personalisierten Managements, was neben differenzialdiagnostischen Einschätzungen und Abgrenzungen auch erforderliche Interventionen bei Funktionseinschränkungen, Komorbiditäten und psychosozialen Problemen umfasst (s. auch QS 5 und 6).

Beim Management von Notfällen geht es zunächst um die Definition - zum einen aus rein medizinischer Sicht und zum anderen aus Patient*innensicht, was sich erheblich unterscheiden kann. Kommt es zu Nervenausfallserscheinungen wegen einer atlantoaxialen Dislokation oder bei neu aufgetretenem Teerstuhl ist die Sache aus ärztlicher Sicht klar, in anderen Fällen hat die erbetene Akutkonsultation aber auch evtl. Zeit bis zur nächsten möglichen Sprechstunde innerhalb eines Werktages, weil der Praxisablauf sonst erschwert werden kann. Weil die akute oder subakute Gesundheitssituation aber am Telefon nicht immer ausreichend eingeschätzt werden kann, ist grundsätzlich eine geregelte Kooperation mit einer akutstationären Einrichtung erforderlich, denn diese hat ja $24 \mathrm{~h} / \mathrm{Tag}$ "geöffnet".

Das Management von Komorbidität ist auf der einen Seite Teil der rheumatologischen Versorgung, da die chronische Entzündung und auch die antirheumati- 


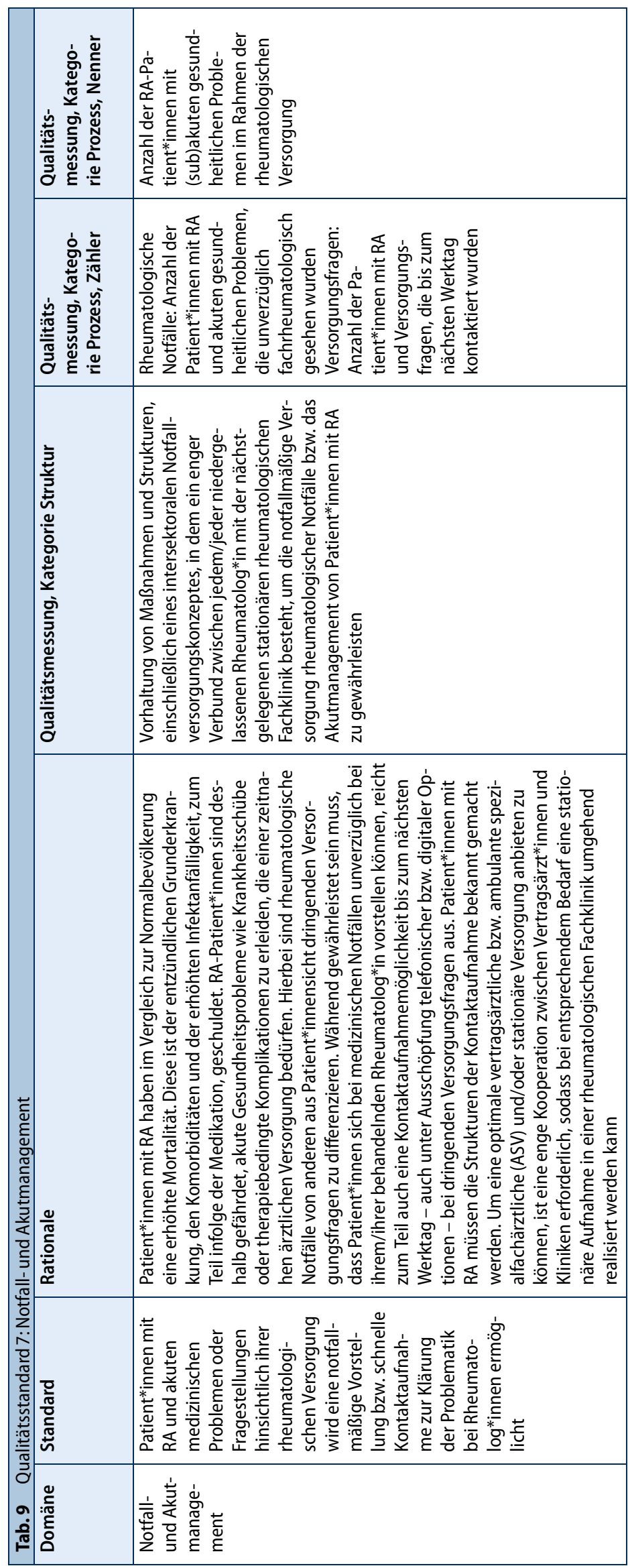

sche Medikation das Risiko für Komorbiditäten erhöhen können, auf der anderen Seite liegt es aber auch in der Verantwortung des Hausarztes. Zum einen sind die bei entzündlich rheumatischen Erkrankungen erhöhte Morbidität und Mortalität v. a. auf kardiovaskuläre Ereignisse zurückzuführen [1,60] und die sowohl durch die Erkrankung als auch durch die Medikation bedingte erhöhte Inzidenz von Osteoporose und dadurch zustande gekommenen Frakturen mit einem erheblichen Leidensdruck der Betroffenen assoziiert [58]. Zum anderen gibt es in Deutschland eine Arbeitsteilung, die eigentlich den Hausärzt*innen die Verantwortung für etablierte kardiovaskuläre Risikofaktoren wie arterielle Hypertonie, Hypercholesterinämie, Diabetes und Rauchen zuweist. Hier sind gezielte Absprachen und gegenseitige Erinnerungen zielführend. Im Rahmen eines jährlichen Assessments können auch Rheumatolog*innen hier initiativ werden und zur Vorbeugung beitragen. Hinsichtlich der Osteoporoseprophylaxe ist eine frühzeitige Einleitung entsprechender Maßnahmen dagegen v.a. bei dem$^{*}$ r Rheumatolog*in als Verordner*in der Osteoporose-fördernden Medikation (Glukokortikoide) anzusiedeln.

Auch die Verordnung von nichtmedikamentösen Therapien wie regelmäßigem Funktionstraining und physiotherapeutischen Maßnahmen sowie die Motivation hin zu mehr körperlicher Aktivität gehören in diesen großen Bereich prophylaktischer und allgemein unterstützender Gesundheitsmaßnahmen.

Am 18.03.2021 wurde vom Gemeinsamen Bundesausschuss (G-BA) beschlossen, ein Disease-Management-Programm (DMP) für die RA in die DMP-Anforderungen-Richtlinien mit aufzunehmen. Dies ist neben der Entwicklung von QS ein guter Schritt in der Verbesserung der Versorgungsqualität der RA-Patient*innen, denn auch hier werden wichtige Schritte der Diagnostik und Therapie in Form von 10 Qualitätszielen definiert, welche sich in großen Teilen mit den hier veröffentlichten QS decken. In Ergänzung zu den hier veröffentlichen QS werden im DMP als Qualitätsziele gesondert nicht rauchende RA-Patient*innen und RA-Patient*innen mit arterieller Hypertonie und einem $\mathrm{RR}<140 / 90 \mathrm{~mm} \mathrm{Hg}$ aufgelistet [17]. 


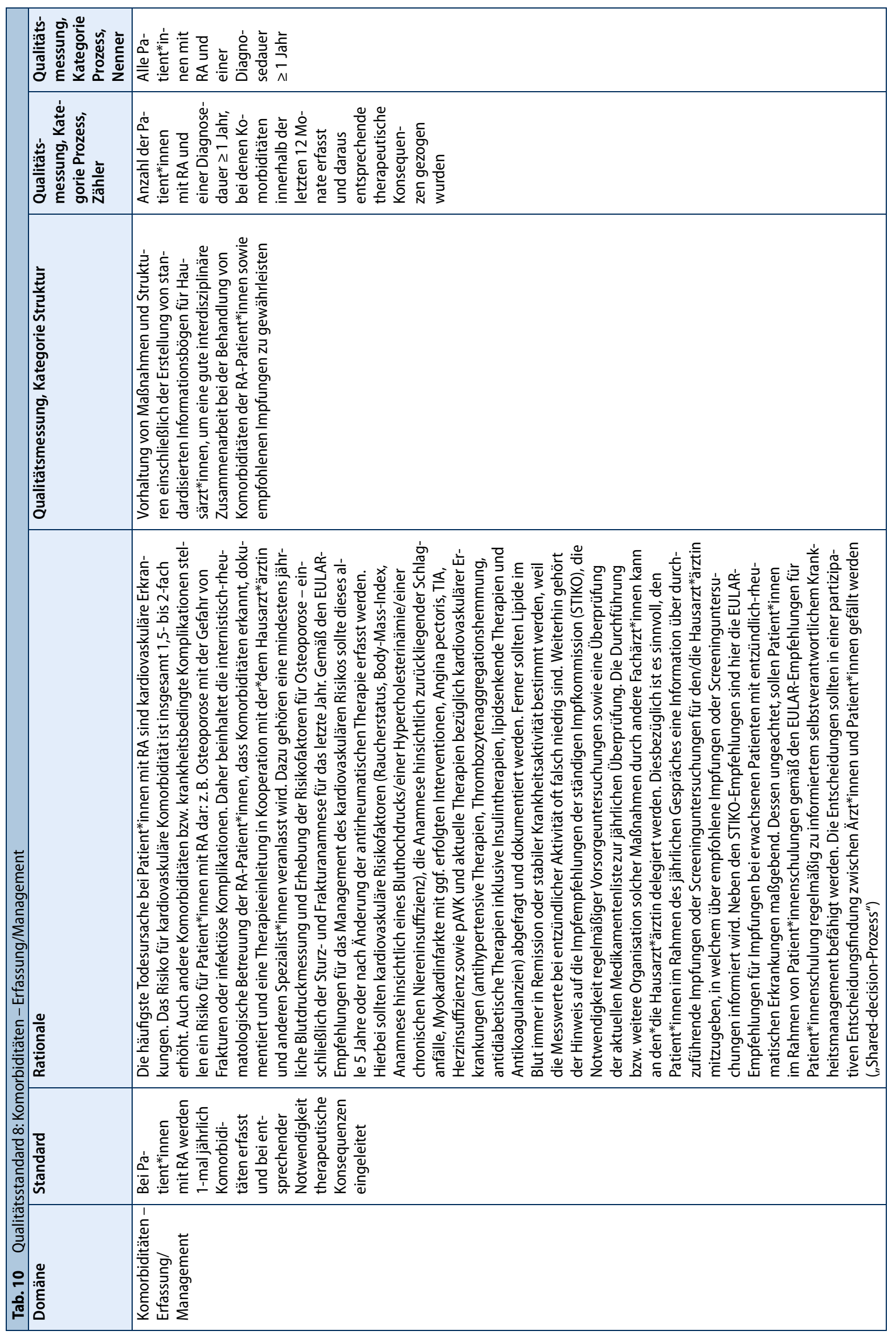


Tab. 11 Grad der Zustimmung zu den einzelnen Qualitätsstandards für Patient*innen mit RA

\begin{tabular}{|l|l|l|l|}
\hline $\begin{array}{l}\text { Qualitäts- } \\
\text { standard }\end{array}$ & Thema & $\begin{array}{l}\text { Zustimmung } \\
\text { ja/nein }\end{array}$ & $\begin{array}{l}\text { Grad der Zustim- } \\
\text { mung (NRS 0-10) }\end{array}$ \\
\hline QS 1 & Frühzeitige Diagnose & $13 / 0$ & $9(2,2)$ \\
\hline QS 2 & Ziel Remission & $13 / 0$ & $9,8(0,4)$ \\
\hline QS 3 & Glukokortikoidfreiheit & $13 / 0$ & $9,3(1,0)$ \\
\hline QS 4 & Konsequente Therapieanpassung & $13 / 0$ & $9,9(0,3)$ \\
\hline QS 5 & $\begin{array}{l}\text { Konsequente Therapie der eingeschränk- } \\
\text { ten Funktionsfähigkeit }\end{array}$ & $13 / 0$ & $9,4(1,1)$ \\
\hline QS 6 & Screening psychosozialer Folgeprobleme & $13 / 0$ & $8,7(1,9)$ \\
\hline QS 7 & Notfall- und Akutmanagement & $13 / 0$ & $9,4(1,0)$ \\
\hline QS 8 & $\begin{array}{l}\text { Komorbiditäten - Erfassung/ } \\
\text { Management }\end{array}$ & $13 / 0$ & $9,2(1,2)$ \\
\hline
\end{tabular}

Zusammengefasst handelt es sich bei den ersten 8 QS für rheumatologische Versorgung um einen ersten wichtigen Meilenstein in Richtung einer systematischen kontinuierlichen Verbesserung der Versorgungsqualität für Patient*innen mit RA in Deutschland. Diese QS fordern eine breite systemisch internistische Versorgung der*s Patient*in von Rheumatolog*innen, die der entzündlichen Erkrankung RA mit all ihren Folgen gerecht wird und sich nicht nur auf die Therapie der betroffenen Gelenke fokussiert. In weiteren Schritten wird zu prüfen sein, wie entsprechende Messungen implementiert und letztlich auch finanziert werden können.

\section{Korrespondenzadresse}

\section{PD Dr. med. U. Kiltz}

Rheumazentrum Ruhrgebiet

Claudiusstr. 45, 44649 Herne, Deutschland uta.kiltz@elisabethgruppe.de

Funding. Open Access funding enabled and organized by Projekt DEAL.

\section{Einhaltung ethischer Richtlinien}

Interessenkonflikt. U. Kiltz, V. Buschhorn-Milberger, K. Albrecht, H.-J. Lakomek, H.-M. Lorenz, M. Rudwaleit, M. Schneider, H. Schulze-Koops, M. Aringer, M. I. Hasenbring, P. Herzer, U. von Hinüber, K. Krüger, A. Lauterbach, B. Manger, R. Oltman, F. Schuch, R. SchmaleGrede, S. Späthling-Mestekemper, S. Zinke und J. Braun geben an, dass kein Interessenkonflikt besteht.

Für diesen Beitrag wurden von den Autoren keine Studien an Menschen oder Tieren durchgeführt. Für die aufgeführten Studien gelten die jeweils dort angegebenen ethischen Richtlinien.

Open Access. Dieser Artikel wird unter der Creative Commons Namensnennung 4.0 International Lizenz veröffentlicht, welche die Nutzung, Vervielfältigung,
Bearbeitung, Verbreitung und Wiedergabe in jeglichem Medium und Format erlaubt, sofern Sie den/die ursprünglichen Autor(en) und die Quelle ordnungsgemäß nennen, einen Link zur Creative Commons Lizenz beifügen und angeben, ob Änderungen vorgenommen wurden.

Die in diesem Artikel enthaltenen Bilder und sonstiges Drittmaterial unterliegen ebenfalls der genannten Creative Commons Lizenz, sofern sich aus der Abbildungslegende nichts anderes ergibt. Sofern das betreffende Material nicht unter der genannten Creative Commons Lizenz steht und die betreffende Handlung nicht nach gesetzlichen Vorschriften erlaubt ist, ist für die oben aufgeführten Weiterverwendungen des Materials die Einwilligung des jeweiligen Rechteinhabers einzuholen.

Weitere Details zur Lizenz entnehmen Sie bitte der Lizenzinformation auf http://creativecommons.org/ licenses/by/4.0/deed.de.

\section{Literatur}

1. Smolen JS, Aletaha D, McInnes IB (2016) Rheumatoid arthritis. Lancet 388(10055):2023-2038

2. Fiehn C, Holle J, Iking-Konert C, Leipe J, Weseloh C, Frerix M et al (2018) S2e guideline: treatment of rheumatoid arthritis with disease-modifying drugs. ZRheumatol 77(Suppl 2):35-53

3. Schneider M, Baseler G, Funken O, Heberger S, Kiltz U, Klose P et al (2020) Management of early rheumatoid arthritis: interdisciplinary guideline. ZRheumatol 79(Suppl 1):1-38

4. Albrecht K, Luque Ramos A, Callhoff J, Hoffmann F Minden K, Zink A (2018) Outpatient care and disease burden of rheumatoid arthritis : Results of a linkage of claims data and a survey of insured persons. ZRheumatol 77(2):102-112

5. Albrecht K, Huscher D, Eidner T, Kleinert S, Spathling-Mestekemper S, Bischoff S et al (2017) Medical treatment of rheumatoid arthritis in 2014: current data from the German collaborative arthritis centers. ZRheumatol 76(1):50-57

6. Albrecht K, Zink A (2008) Versorgungssituation der rheumatoiden Arthritis in Deutschland. Akt Rheumatol 43:369-374

7. Albrecht K, Luque Ramos A, Hoffmann F, Redeker I, Zink A (2018) High prevalence of diabetes in patients with rheumatoid arthritis: results from a questionnaire survey linked to claims data. Rheumatology (Oxford) 57(2):329-336
8. Jacobs H, Callhoff J, Hoffmann F, Zink A, Albrecht K (2019) Non-drug treatment of rheumatoid arthritis: an analysis of claims data and a survey of insured persons (Project PROCLAIR). Z Rheumatol 78(2):119-126

9. Albrecht K, Huscher D (2017) Do we prescribe physical medicine sufficiently? Current data from the German Collaborative Arthritis Center. Akt Rheumatol 42:118-121

10. Albrecht K, Callhoff J, Zink A (2019) Long-term trends in rheumatology care: achievements and deficits in 25 years of the German national rheumatology database. ZRheumatol 78(8):703-712

11. Luque Ramos A, Redeker I, Hoffmann F, Callhoff J, Zink A, Albrecht K (2019) Comorbidities in patients with rheumatoid arthritis and their association with patient-reported outcomes: results of claims data linked to questionnaire survey. J Rheumatol 46(6):564-571

12. Lindner $\mathrm{L}$, Callhoff J, Alten R, Krause A, Ochs W, Zink A et al (2020) Osteoporosis in patients with rheumatoid arthritis: trends in the German National Database 2007-2017. Rheumatol Int 40(12):2005-2012

13. Kiltz U, Celik A, Tsiami S, Buehring B, Baraliakos X, Andreica I et al (2021) Are patients with rheumatic diseases on immunosuppressive therapies protected against preventable infections? A crosssectional cohort study. RMD Open 7(1):e1499

14. Luque Ramos A, Hoffmann F, Callhoff J, Zink A, Albrecht K (2016) Influenza and pneumococcal vaccination in patients with rheumatoid arthritis in comparison with age- and sex-matched controls: results of a claims data analysis. Rheumatol Int 36(9):1255-1263

15. Luque Ramos A, Albrecht K, Zink A, Hoffmann F (2017) Rheumatologic care of nursing home residents with rheumatoid arthritis: a comparison of the year before and after nursing home admission. Rheumatol Int 37(12):2059-2064

16. Schwarze M, Fieguth V, Schuch F, Sandner P, Edelmann E, Handel A et al (2021) Disease-related knowledge acquisition through structured patient information in rheumatoid arthritis (StruPI-RA): first results of the StruPI-RA study in Germany. ZRheumatol 80(4):364-372

17. Gemeinsamer Bundesausschuss (2021) Beschluss des Gemeinsamen Bundesausschusses über die 26. Änderung der DMP-Anforderungen-Richtlinie (DMP-A-RL): Änderung der Anlage 2, Ergänzung der Anlage 21 (DMP Rheumatoide Arthritis) und der Anlage 22 (Rheumatoide Arthritis Dokumentation)

18. Solomon DH, Gabriel SE (2007) Quality measures 101: what every rheumatologist should know. Clin Exp Rheumatol 25(6Suppl 47):18-21

19. National Institute for Health and Care Excellence (Nice) (2016) (Nice) NlfHaCE. Quality standards process guide. https://www.nice.org.uk/ standards-and-indicators/timeline-developingquality-standards.Zugegriffen:02.03.2021

20. Kiltz $U$, Landewe RBM, van der Heijde $D$, Rudwaleit $\mathrm{M}$, Weisman $\mathrm{MH}$, Akkoc $\mathrm{N}$ et al (2020) Development of ASAS quality standards to improve the quality of health and care services for patients with axial spondyloarthritis. Ann Rheum Dis 79(2):193-201

21. Donabedian A (1980) Methods for deriving criteria for assessing the quality of medical care. Med Care Rev 37(7):653-698

22. Lakomek HJ, Rudwaleit $M$, Hentschel $A$, Broge $B$, Abrolat J, Bessler F et al (2021) Quality in acute inpatient rheumatology 2021: Current aspects of the KOBRA quality label of the Association of 
Rheumatological Acute Care Clinics. Z Rheumatol 80(8):758-770

23. Roeder N, Lakomek HJ (2011) The "outcome benchmarking in rheumatologic acute care" project of the Association of Rheumatologic Acute Care Clinics (VRA e. V.) in Germany. Z Evid Fortbild Qual Gesundhwes 105(5):343-349

24. Lakomek HJ, Braun J, Gromnica-Ihle E, Fiehn C, Claus S, Specker C et al (2011) Amendment of the structural quality for inpatient rheumatology. A forward-looking concept. Z Rheumatol 70(7):615-619

25. Kiltz U, Buschhorn-Milberger V, Albrecht $\mathrm{K}$ et al (2021) Entwicklung von Qualitätsstandards für Patient*innen mit axialer Spondyloarthritis zum Einsatz in Deutschland. Z Rheumatol. https://doi. org/10.1007/s00393-021-01019-x

26. Macieira C, Cunha-Miranda L, Nero P, Laires $P$, Bogas M, Farinha $S$ et al (2019) Rheuma SPACE-Standard Practice Aiming Clinical Excellence: description of the methodological approach. Acta Reumatol Port 46(2):140-155

27. Yazdany J, Robbins M, Schmajuk G, Desai S, Lacaille D, Neogi T et al (2016) Development of the American college of rheumatology's rheumatoid arthritis electronic clinical quality measures. Arthritis Care Res (Hoboken) 68(11):1579-1590

28. Ivorra JA, Martinez JA, Lazaro $P$, Navarro $F$, Fernandez-Nebro A, de Miguel E et al (2013) Quality-of-care standards for early arthritis clinics. Rheumatol Int 33(10):2459-2472

29. van Hulst LT, Fransen J, den Broeder AA, Grol R, van Riel PL, Hulscher ME (2009) Development of quality indicators for monitoring of the disease course in rheumatoid arthritis. Ann Rheum Dis 68(12):1805-1810

30. Kennedy T, McCabe C, Struthers G, Sinclair H, Chakravaty K, Bax D et al (2005) BSR guidelines on standards of care for persons with rheumatoid arthritis. Rheumatology (Oxford) 44(4):553-556

31. Petersson IF, Strombeck B, Andersen L, Cimmino M, Greiff R, Loza E et al (2014) Development of healthcare quality indicators for rheumatoid arthritis in Europe: the eumusc.net project. Ann Rheum Dis 73(5):906-908

32. Barber CE, Marshall DA, Mosher DP, Akhavan $P$, Tucker L, Houghton K et al (2016) Development of system-level performance measures for evaluation of models of care for inflammatory arthritis in Canada. JRheumatol 43(3):530-540

33. Kiltz $U$, Landewe RBM, van der Heijde $D$, Rudwaleit $\mathrm{M}$, Weisman $\mathrm{MH}$, Akkoc $\mathrm{N}$ et a (2019) Development of ASAS quality standards to improve the quality of health and care services for patients with axial spondyloarthritis. Ann Rheum Dis 79(2):193-201

34. Johansen I, Klokkerud M, Anke A, Borke JB, Glott T, Hauglie U et al (2019) A quality indicator set for use in rehabilitation team care of people with rheumatic and musculoskeletal diseases; development and pilot testing. BMC Health Serv Res 19(1):265

35. Uhlig T, Lie E, Norvang V, Lexberg AS, Rodevand E, Kroll $F$ et al (2016) Achievement of remission and low disease activity definitions in patients with rheumatoid arthritis in clinical practice: results from the NOR-DMARD study. J Rheumatol 43(4):716-723

36. Norvang V, Brinkmann GH, Yoshida K, Lillegraven $S$, Aga AB, Sexton J et al (2020) Achievement of remission in two early rheumatoid arthritis cohorts implementing different treat-to-target strategies. Arthritis Rheumatol 72(7):1072-1081

\section{Development of quality standards for patients with rheumatoid arthritis for use in Germany}

Despite a qualitatively and structurally good care of patients with rheumatoid arthritis (RA) in Germany, there are still potentially amendable deficits in the quality of care. For this reason, the German Society for Rheumatology (DGRh) has therefore decided to ask a group of experts including various stakeholders to develop quality standards (QS) for the care of patients with RA in order to improve the quality of care. The QS are used to determine and quantitatively measure the quality of care, subject to relevance and feasibility. The recently published NICE and ASAS standards and a systematic literature search were used as the basis for development. A total of 8 QS, now published for the first time, were approved with the intention to measure and further optimize the quality of care for patients with RA in Germany.

\section{Keywords}

Rheumatoid arthritis · Quality of care · Quality standards · Care gaps

37. Kapral T, Dernoschnig F, Machold KP, Stamm T, Schoels M, Smolen JS et al (2007) Remission by composite scores in rheumatoid arthritis: are ankles and feet important? Arthritis Res Ther 9(4):R72

38. Studenic P, Smolen JS, Aletaha D (2012) Near misses of ACR/EULAR criteria for remission: effects of patient global assessment in Boolean and index-based definitions. Ann Rheum Dis 71(10):1702-1705

39. Duru N, van der Goes MC, Jacobs JW, Andrews T, Boers M, Buttgereit F et al (2013) EULAR evidencebased and consensus-based recommendations on the management of medium to high-dose glucocorticoid therapy in rheumatic diseases. Ann Rheum Dis 72(12):1905-1913

40. Ferreira RJO, Carvalho PD, Ndosi M, Duarte C, Chopra A, Murphy E et al (2019) Impact of patient global assessment on achieving remission in patients with rheumatoid arthritis: a multinational study using the METEOR database. Arthritis Care Res (Hoboken) 71(10):1317-1325

41. Paulshus Sundlisaeter $N$, Olsen IC, Aga AB, Hammer HB, Uhlig T, van der Heijde D et al (2018) Predictors of sustained remission in patients with early rheumatoid arthritis treated according to an aggressive treat-to-target protocol. Rheumatology (Oxford) 57(11):2022-2031

42. Westhoff G, Listing J, Zink A (2000) Loss of physical independence in rheumatoid arthritis: interview data from a representative sample of patients in rheumatologic care. Arthritis Care Res 13(1):11-22

43. Krishnan E, Sokka T, Hakkinen A, Hubert $H$, Hannonen P (2004) Normative values for the Health Assessment Questionnaire disability index: benchmarking disabilityin the general population. Arthritis Rheum 50(3):953-960

44. Leblanc-Trudeau C, Dobkin PL, Carrier N, CossetteP, de Brum-Fernandes AJ, Liang $P$ et al (2015) Depressive symptoms predict future simple disease activity index scores and simple disease activity index remission in a prospective cohort of patients with early inflammatory polyarthritis. Rheumatology (Oxford) 54(12):2205-2214

45. Sparks JA, Chang SC, Liao KP, Lu B, Fine AR, Solomon DH et al (2016) Rheumatoid arthritis and mortality among women during 36 years of prospective follow-up: results from the nurses' health study. Arthritis Care Res (Hoboken) 68(6):753-762
46. Agca R, Heslinga SC, Rollefstad S, Heslinga M, McInnes IB, Peters MJ et al (2017) EULAR recommendations for cardiovascular disease risk management in patients with rheumatoid arthritis and other forms of inflammatory joint disorders: 2015/2016 update. Ann Rheum Dis 76(1):17-28

47. Lakomek HJ, Neeck G, Lang B, Jung J (2002) Structural quality of acute internal medicine rheumatology clinics-Project Group of the Association of Rheumatologic Acute Clinics. ZRheumatol 61(4):405-414

48. Lakomek HJ, Hulsemann JL, Kuttner T, Buscham K, Roeder N (2007) Clinical pathways in rheumatological inpatient treatment-A structured process management.ZRheumatol 66(3):247-254

49. Zink A, Braun J, Gromnica-Ihle E, Krause D, Lakomek HJ, Mau W et al (2017) Memorandum of the German Society for Rheumatology on the quality of treatment in rheumatology-Update 2016.ZRheumatol 76(3):195-207

50. Braun J, Krause A, Aringer M, Burmester G, Bessler F, Engel JM et al (2016) Standards of care for people with rheumatoid arthritis in Europe: Translation and comments of the eumusc.net recommendations supported by EULAR performed by a national task force of the professional organisations DGRh and VRA supported by "Deutsche Rheumaliga". ZRheumatol 75(4):416-428

51. Lakomek HJ, Bungard S, Rudwaleit M, Bessler F, Braun J, Fiehn C, Gromnica-Ihle E, Hellmich B, Kneitz C, Krause A, Veit C, Fiori W, Roeder N (2014) Das „KOBRA-Qualitätsprojekt“ - Verleihung eines Qualitätssiegels an Einrichtungen der akutstationären Rheumatologie. Krankenhaus 106(10):914-923

52. Fiori W, Lakomek HJ, Liman W, Lenz M, Hochreutener MA, Roeder N (2009) Qualität verbessern durch Benchmarking. Arthritis Rheuma 29:371-377

53. Lorenz HM, Aringer M, Braun J, Hoyer BF, Krause A, Meyer-Olson D et al (2021) Mission statement from rheumatologists in the German Society of Rheumatology (DGRh e. V.): We live rheumatology. ZRheumatol 80(Suppl 1):10-12

54. Edelmann E, Lakomek HJ, Bessler F, Braun J, Froschauer S, Hellmich B et al (2019) Outpatient specialist medical treatment (ASV) - A new treatment level in rheumatology. Z Rheumatol 78(8):765-773 
55. Felson DT, Smolen JS, Wells G, Zhang B, van Tuyl LH, Funovits J et al (2011) American College of Rheumatology/European League against Rheumatism provisional definition of remission in rheumatoid arthritis for clinical trials. Ann Rheum Dis 70(3):404-413

56. Albrecht K, Callhoff J, Edelmann E, Schett G, Schneider M, Zink A (2016) Clinical remission in rheumatoid arthritis. Data from the early arthritis cohort study CAPEA. ZRheumatol 75(1):90-96

57. Schwarting A, Dreher M, Assmann G, Witte T, Hoeper K, Schmidt RE (2019) Experiences and results from Rheuma-VOR. ZRheumatol 78(8):743-752

58. Buckley L, Humphrey MB (2018) Glucocorticoid-induced osteoporosis. N Engl J Med 379(26):2547-2556

59. Wassenberg S, Rau R, Steinfeld P, Zeidler H (2005) Very low-dose prednisolone in early rheumatoid arthritis retards radiographic progression over two years: a multicenter, double-blind, placebocontrolled trial. Arthritis Rheum 52(11):3371-3380

60. Jafri K, Bartels CM, Shin D, Gelfand JM, Ogdie A (2017) Incidence and management of cardiovascular risk factors in psoriatic arthritis and rheumatoid arthritis: a population-based study. Arthritis Care Res (Hoboken) 69(1):51-57

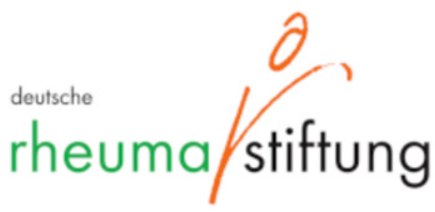

rheuma heilbar machen

\section{Deutsche Rheumastiftung verleiht wissenschaftliche Nachwuchs- und Förderpreise 2022}

Anlässlich des 50. Deutschen Rheumatologiekongresses vom 31. 08. bis zum 02.09. 2022 in Berlin würdigte die Deutsche Rheumastiftung besondere Leistungen für das Fachgebiet und zeichnete Fachärztinnen und Fachärzte in den Kategorien Ideenwettbewerb, Carol Nachman Nachwuchspreis und Projektpreisen aus.

Im Rahmen eines wissenschaftlichen Symposiums am 2. September zeichnete die Deutsche Rheumastiftung (DRS) insgesamt sieben Kolleginnen und Kollegen des Faches Rheumatologie für innovative wissenschaftliche Arbeiten und Forschungen aus:

Den mit 5.000 Euro dotierten Carol-Nachman-Nachwuchs-Forschungspreis erhielt Dr. med. Ricardo Grieshaber Bouyer aus Heidelberg für seine Forschungsarbeit The neutrotime transcriptional signature defines a single continuum of neutrophils across biological compartments.

Gleich drei Mal würdigte die DRS die Preisträgerinnen und Preisträger des diesjährigen Ideenwettbewerbes. Jeweils 5.000 Euro erhielten als Fördersumme Dr. med. Isabell Haase aus Düsseldorf für Ihre Arbeit Rheuma und Partnerschaft; Dr. med. Thomas Schneidereit aus Wuppertal zu seinen Forschungen Früherkennung einer Alveolitis bei mit systemischer Sklerose assoziierten interstitiellen Lungenerkrankung durch Messung der alveolären NO-Konzentration zuhause und Dr. med. Tatjana Welzel mit Prof. und Dr. med. Kirsten Minden aus Berlin gemeinschaftlich für die wissenschaftliche Arbeit zur Verbesserung der Gesundheit von Kindern und Jugendlichen mit autoinflammatorischen Erkrankungen (AID).

Zum ersten Mal verlieh die Deutsche Rheumastiftung Projektpreise mit einer Gesamtdotierung von 40.000 Euro. Insgesamt erhielten vier Preisträgerinnen jeweils 10.000 Euro Preisgeld als Förderung. Für ihre Arbeit Understanding and using health information in Rheumatology. Wie suchen, finden und nutzen Patienten mit entzündlich-rheumatischen Erkrankungen (ERE) webbasierte Krankheitsinformationen im Alltag? wurde Dr. med. Iona Andreica aus Herne ausgezeichnet. Dr. med. Christina Düsing aus Düsseldorf erhielt für Erstellen eines Flyers mit Empfehlungen zur Umsetzung der Ergebnisse der von der Rheuma-Liga geförderten Studie "Fatigue bei Patient:innen, für den klinischen Gebrauch den Projektpreis. PD Dr. Uta Kiltz aus Herne wurde gewürdigt für ihre Darlegung der Faktoren im Zusammenhang mit Suizidgedanken bei Patienten mit entzündlichrheumatischen Erkrankungen (SARA Projekt). Für ihre Untersuchung der $\beta 2$-adrenergen Rezeptors Polymorphismen bei Patienten mit rheumatoider Arthritis als potentieller genetischer Risikofaktor der Fatigue wurde PD Dr. Olga Seifert aus Leipzig gewürdigt.

Zwei der vier Projektpreise wurden an Bewerber verliehen, die sich gemeinsam mit dem „Patient Partner Projekt" der Deutschen Rheuma-Liga beworben hatten. „Forschung für Menschen mit rheumatischen Erkrankungen, aber auch zusammen mit Rheumakranken Menschen - Das ist wirklich etwas Einmaliges!" so der Rheumatologe Dr. Florian Schuch, Erlangen, Vorstand der Deutschen Rheumastiftung.

Der nächste Kongress findet vom 30 . August bis 02 . September 2023 in Leipzig statt.

Weitere Informationen: www.deutsche-rheumastiftung.de https://dgrh-kongress.de/ 